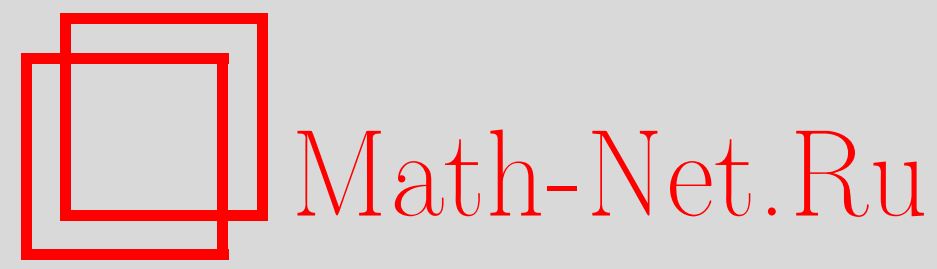

F. den Hollander, S. Whittington, Localization transition for a copolymer in an emulsion, Теория вероятн. и ее примен., 2006, том 51, выпуск 1, 193240

DOI: https://doi.org/10.4213/tvp154

Использование Общероссийского математического портала MathNet.Ru подразумевает, что вы прочитали и согласны с пользовательским соглашением

http://www . mathnet.ru/rus/agreement

Параметры загрузки:

IP : 52.205 .19 .152

26 апреля 2023 г., 18:19:24

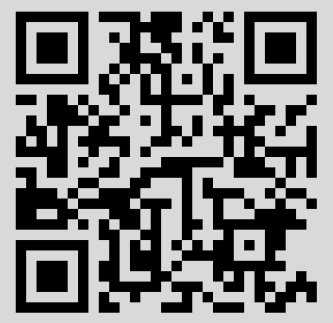


(C) 2006 r. DEN HOLLANDER F.*, WHITTINGTON S. G.**

\section{LOCALIZATION TRANSITION FOR A COPOLYMER IN AN EMULSION}

В этой статье мы изучаем двумерные направленные несамопересекающиеся случайные блуждания, моделирующие кополимеры в случайных растворах. Полимер состоит из последовательно соединенных мономеров двух типов $A$ и $B$, каждый из которых встречается с вероятностью $\frac{1}{2}$. Раствор представляет собой случайную смесь жидкостей двух типов, $A$ и $B$, объединенных в большие квадратные блоки, встречаюшиеся с вероятностью $p$ и $1-p$ соответственно, $p$ изменяется от 0 до 1 . Полимер в растворе имеет энергию, равную $-\alpha$, умноженное на число $A A$-пар плюс $-\beta$, умноженное на число $B B$-пар, где $\alpha, \beta$ - параметры взаимодействия. Соображения симметрии показывают, что без потери общности мы можем ограничиться рассмотрением конуса $\{(\alpha, \beta): \alpha \geqslant|\beta|\}$.

Мы выводим вариационное выражение для суммарной свободной энергии/мономер в пределе, когда длина полимера стремится к бесконечности и блоки в растворе имеют размеры $L_{n}$ так, что $L_{n}$ стремится к бесконечности, а отношение $L_{n} / n$ стремится к нулю. Для того чтобы изучить модель математически, мы предполагаем, что полимер может входить и выходить из раствора через пару соседних блоков в диаметрально противоположных углах. Хотя это ограничение не имеет физического смысла, модель обнаруживает богатое и физически осмысленное поведение.

Пусть $p_{c}$, приблизительно равное 0.64 , есть критическая вероятность для направленной проблемы просачивания на квадратной решетке. Мы показываем, что при $p \geqslant p_{c}$ свободная энергия имеет фазовый переход вдоль кривой в конусе, который оказывается независимым от $p$. Для $p<p_{c}$ мы показываем, что свободная энергия имеет фазовый переход вдоль двух кривых в конусе, зависящий от $p$. Мы получаем ряд результатов о качественных свойствах этих кривых.

Ключевые слова и фразы: случайный кополимер, случайный раствор, локализация, делокализация, фазовый переход, просачивание, большие уклонения.

* Mathematical Institute, Leiden University, P.O. Box 9512, 2300 RA Leiden, The Netherlands; EURANDOM, P.O. Box 513, 5600 MB Eindhoven, The Netherlands; e-mail: denholla@math.leidenuniv.nl

** Department of Chemistry, University of Toronto, Toronto, Canada M5S 3H6 e-mail: swhittin@chem.utoronto.ca 


\section{Introduction and main results}

1.1. Background. (Linear) copolymers are polymer chains consisting of two or more types of monomer. Random copolymers are copolymers where the order of the monomers along the polymer chain is determined by a random process. In any particular chain, the sequence of monomers once determined is fixed, so a random copolymer is an example of a quenched random system. In this paper we will be concerned with copolymers consisting of two types of monomer, labelled $A$ and $B$. We write $\omega_{i} \in\{A, B\}$ to denote the type of the $i$-th monomer, and $\omega=\left\{\omega_{1}, \omega_{2}, \cdots\right\}$ to denote the full order along the chain, which is truncated at $\omega_{n}$ when the polymer has length $n$. We will only consider the case where the random variables $\omega_{i}$ are independent and identically distributed (i.i.d.), assuming the values $A$ and $B$ with probability $\frac{1}{2}$ each. In principle, the properties of the polymer depend on $\omega$, and we write $P_{n}^{\omega}$ for the value of a property $P$ when the polymer has length $n$ and order $\omega$. If $\lim _{n \rightarrow \infty} P_{n}^{\omega}$ exists $\omega$-a.s. and is nonrandom, then we say that the property $P$ is self-averaging.

Several different physical situations are of interest. For instance, if the monomer-monomer interactions differ for pairs $A A, B B$, and $A B$, then we may investigate the effect of the randomness on the collapse transition, where the polymer collapses from a random coil to a ball-like structure as the temperature decreases or the solvent quality varies. Alternatively, if the two types of monomer interact differently with an impenetrable surface, then we may investigate the effect of the randomness on the adsorption transition, where the polymer adsorbs onto the surface as the temperature decreases or the surface quality varies. There are interesting questions about how the location of the collapse transition or the adsorption transition, and the values of associated critical exponents, depend on the parameters controlling the randomness. Many of these questions remain unresolved. For background and references, the reader is referred to [26], [27], [19], [5], [33], and [30].

The problem that we will consider here is the localization transition of a random copolymer near an interface. Suppose that we have two immiscible liquids and that it is energetically favorable for monomers of one type to be in one liquid and for monomers of the other type to be in the other liquid. At high temperatures the polymer will delocalize into one of the liquids in order to maximize its entropy, while at low temperatures energetic effects will dominate and the polymer will localize close to the interface between the two liquids in order to be able to place more than half of its monomers in their preferred liquid. In the limit as $n \rightarrow \infty$, we may expect a phase transition. A typical example here would be an oil-water interface and a copolymer with hydrophobic and hydrophilic monomers.

Given such a physical situation, the polymer can be modelled in a variety of ways, e.g., as a random walk or as a self-avoiding walk, either directed or 
undirected. Such examples have been investigated for the situation where the interface is flat and infinite. In addition, there is some flexibility in the details of the Hamiltonian that is chosen to model the interactions.

A simple model with a single interface was proposed and analyzed by Garel et al [9], with a Hamiltonian that depends on temperature and interaction bias. A first mathematical treatment of this model was given by Sinai [29] and by Grosberg et al [15], in the absence of interaction bias, for a directed random walk version of the model. For this version, Bolthausen and den Hollander [4] proved that the quenched free energy is nonanalytic along a critical curve in the plane of inverse temperature vs. interaction bias, and derived several qualitative properties of this curve, among which upper and lower bounds. Albeverio and Zhou [1], for the unbiased case, and Biskup and den Hollander [2], for the biased case, extended this work by deriving path properties of the model, in particular, ergodic limits along the interface and exponential tightness perpendicular to the interface in the localized phase, as well as zero limiting frequency of hits of the interface in the interior of the delocalized phase. The latter result was recently strengthened by Giacomin and Toninelli [11], who showed that in the interior of the delocalized phase the number of times the path intersects the interface grows at most logarithmically with its length. The conjecture is that the number of intersections is actually bounded. Giacomin and Toninelli in [13] proved that the free energy is infinitely differentiable inside the localized phase. Thus, there is no phase transition of finite order anywhere off the critical curve. Moreover, in [12] they proved that the free energy is twice differentiable across the critical curve, i.e., the phase transition is at least of second order.

Maritan et al [22] considered both random walk and self-avoiding walk models and derived rigorous bounds on the free energy, under an assumption on the asymptotics of a certain class of self-avoiding walks. Martin et al [23] proved the existence of a localization transition for a self-avoiding walk model and obtained qualitative results about the shape of the phase transition curve. These results were extended and improved by Madras and Whittington [21], who also gave a rigorous version of the result of Maritan et al [22] for the self-avoiding walk model. Orlandini et al [25] derived rigorous bounds on the critical curve for the directed random walk model, while Causo and Whittington [7] and James et al [18] obtained sharp numerical estimates for the self-avoiding walk model.

An interesting recent development concerns the slope of the critical curve in the limit of small inverse temperature and interaction bias in the directed random walk version of the model. In [4] it was proved that this slope exists, is strictly positive and is at most 1 , the latter being a corollary of an upper bound on the full critical curve. Garel et al [9] had earlier hinted at the possibility that this slope be 1 , a viewpoint that was taken up by Trovato and Maritan [32]. However, Stepanow et al [31] conjectured 
the slope to be $\frac{2}{3}$, based on replica symmetry arguments. Monthus [24], using a general renormalization scheme, conjectured a simple explicit formula for the full critical curve, which indeed has slope $\frac{2}{3}$ in the limit of small inverse temperature and interaction bias. Based on this work, Bodineau and Giacomin [3] proved that this formula is a lower bound for the critical curve, so that we now know that the slope is at least $\frac{2}{3}$. Numerical work by Garel and Monthus [10] and Caravenna et al [6] indicates that the upper and lower bounds on the critical curve are not sharp, nor are the bounds 1 and $\frac{2}{3}$ for the slope. So far all attempts to improve these bounds have failed. The slope seems to be close to 0.82 .

The reason why the above issue is of interest is that, while the full shape of the critical curve is model-dependent, the slope in the limit of small inverse temperature and interaction bias is believed to be insensitive to the details of the model.

The goal of the present paper is to study a model where the interface has a more complex geometry. A first attempt in this direction was made by den Hollander and Wüthrich [17], where an infinite array of parallel flat infinite interfaces was considered and the average hopping time between interfaces was computed for a directed random walk model. In the present paper we investigate the situation in which the lattice is divided into large blocks, and each block is independently labelled $A$ or $B$ with probability $p$ and $1-p$, respectively, i.e., the interface has a percolation type structure. This is a primitive model of an emulsion (e.g., oil dispersed as droplets in water as the dispersing medium). As before, the copolymer consists of a random concatenation of monomers of type $A$ and $B$. It is energetically favorable for monomers of type $A$ to be in the $A$-blocks and for monomers of type $B$ to be in the $B$-blocks of the emulsion. Under the restriction that the polymer can only enter and exit a pair of neighboring blocks at diagonally opposite corners, we show that there is a phase transition between a phase where the polymer is fully delocalized away from the interfaces between the two types of blocks and a phase where the polymer is partially localized near the interfaces. It turns out that the critical curve does not depend on $p$ in the supercritical percolation regime, but does depend on $p$ in the subcritical percolation regime. In the latter regime, a second critical curve appears separating two partially localized phases.

Our paper is organized as follows. In the rest of Section 1 we define the model, formulate our main theorems, discuss these theorems, and formulate some open problems. Section 2 contains some preparatory results about path entropies and free energies per pair of neighboring blocks. In Sections 3 and 4 we provide the proofs of the main theorems, focussing on the free energy, respectively, the critical curves.

1.2. The model. Each positive integer is randomly labelled $A$ or $B$, with probability $\frac{1}{2}$ each, independently for different integers. The resulting 
labelling is denoted by $\omega=\left\{\omega_{i}: i \in \mathbf{N}\right\} \in\{A, B\}^{\mathbf{N}}$. Fix $p \in(0,1)$ and $L_{n} \in \mathbf{N}$. Partition $\mathbf{R}^{2}$ into square blocks of size $L_{n}$ :

$$
\mathbf{R}^{2}=\bigcup_{x \in \mathbf{Z}^{2}} \Lambda_{L_{n}}(x), \quad \Lambda_{L_{n}}(x)=x L_{n}+\left(0, L_{n}\right]^{2} .
$$

(Note that the blocks contain their north and east side but not their south and west side.) Each block is randomly labelled $A$ or $B$, with probability $p$, respectively, $1-p$, independently for different blocks. The resulting labelling is denoted by

$$
\Omega=\left\{\Omega(x): x \in \mathbf{Z}^{2}\right\} \in\{A, B\}^{\mathbf{Z}^{2}} .
$$

Consider the set of $n$-step directed self-avoiding paths starting at the origin and being allowed to move upwards, downwards, and to the right. Let $\mathscr{W}_{n, L_{n}}$ be the subset of those paths that enter blocks at a corner, exit blocks at one of the two corners diagonally opposite the one where it entered, and in between stay confined to the two blocks that are seen when entering. In other words, after the path reaches a site $x L_{n}$, it must make a step to the right, it must subsequently stay confined to the pair of blocks labelled $x$ and $x+(0,-1)$, and it must exit this pair of blocks either at site $x L_{n}+\left(L_{n}, L_{n}\right)$ or at site $x L_{n}+\left(L_{n},-L_{n}\right)$ (see Figure 1). This restriction is put in to make the model mathematically tractable.

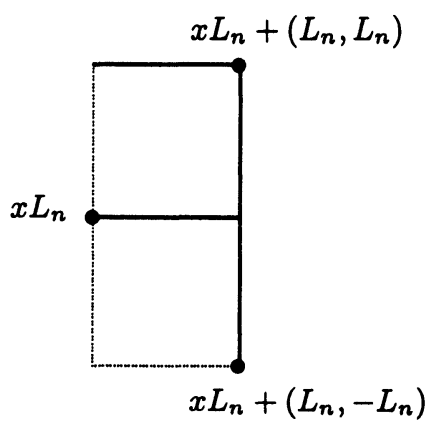

Fig. 1. Two neighboring blocks. The dots are the sites of entrance and exit. The drawn lines are part of the blocks, the dashed lines are not.

Given $\omega, \Omega$, and $n$, with each path $\pi \in \mathscr{W}_{n, L_{n}}$ we associate an energy given by the Hamiltonian

$$
H_{n, L_{n}}^{\omega, \Omega}(\pi)=-\sum_{i=1}^{n}\left(\alpha 1\left\{\omega_{i}=\Omega_{\pi_{i}}^{L_{n}}=A\right\}+\beta 1\left\{\omega_{i}=\Omega_{\pi_{i}}^{L_{n}}=B\right\}\right),
$$

where $\pi_{i}$ denotes the $i$-th step of the path and $\Omega_{\pi_{i}}^{L_{n}}$ denotes the label of the block that step $\pi_{i}$ lies in. What this Hamiltonian does is count the number of $A A$-matches and $B B$-matches and assign them energy $-\alpha$ and $-\beta$, respectively, where $\alpha, \beta \in \mathbf{R}$. Note that the interaction is assigned to bonds rather than to sites: We identify the monomers with the steps of the path. 
For $\alpha, \beta>0$, the above definitions are to be interpreted as follows: $\omega$ plays the role of the random monomer types, with $A$ denoting hydrophobic and $B$ denoting hydrophilic; $\Omega$ plays the role of the random emulsion, with $A$ denoting oil and $B$ denoting water; $n$ is the number of monomers; the Hamiltonian assigns negative energy to matches of affinities between polymer and emulsion, with $\alpha$ and $\beta$ the interaction strengths (it assigns zero energy to mismatches).

Given $\omega, \Omega$, and $n$, we define the quenched free energy per step as

$$
f_{n, L_{n}}^{\omega, \Omega}=\frac{1}{n} \ln Z_{n, L_{n}}^{\omega, \Omega}, \quad Z_{n, L_{n}}^{\omega, \Omega}=\sum_{\pi \in \mathscr{W}_{n, L_{n}}} \exp \left[-H_{n, L_{n}}^{\omega, \Omega}(\pi)\right] .
$$

We are interested in the limit as $n \rightarrow \infty$ subject to the restriction

$$
L_{n} \rightarrow \infty \text { and } \frac{L_{n}}{n} \rightarrow 0 .
$$

This is a coarse-graining limit where the path spends a long time in each single block yet visits many blocks. Throughout the paper we assume that this restriction is in force, which is necessary to make the model mathematically tractable. It will turn out that the free energy does not depend on the choice of the sequence $\left(L_{n}\right)_{n \in \mathbf{N}}$.

1.3. Free energy. Theorem 1.3 .1 below says that the quenched free energy per step is self-averaging and can be expressed as a variational problem involving the free energies of the polymer in each of the four possible pairs of adjacent blocks it may encounter and the frequencies at which the polymer visits each of these pairs of blocks on the coarse-grained block scale. To formulate this theorem we need some more definitions.

First, for $L \in \mathbf{N}$ and $a \geqslant 2$ (with $a L$ integer), let $\mathscr{W}_{a L, L}$ denote the set of $a L$-step directed self-avoiding paths starting at $(0,0)$, ending at $(L, L)$, and in between not leaving the two adjacent blocks of size $L$ labelled $(0,0)$ and $(-1,0)$. For $k, l \in\{A, B\}$, let

$$
\psi_{k l}^{\omega}(a L, L)=\frac{1}{a L} \ln Z_{a L, L}^{\omega}, \quad Z_{a L, L}^{\omega}=\sum_{\pi \in \mathscr{W}_{a L, L}} \exp \left[-H_{a L, L}^{\omega, \Omega}(\pi)\right]
$$

when $\Omega(0,0)=k$ and $\Omega(0,-1)=l$, denote the free energy per step in a $k l$-block when the number of steps inside the block is $a$ times the size of the block. Let

$$
\lim _{L \rightarrow \infty} \psi_{k l}^{\omega}(a L, L)=\psi_{k l}(a)=\psi_{k l}(\alpha, \beta ; a)
$$

Note here that $k$ labels the type of the block that is diagonally crossed, while $l$ labels the type of the block that appears as its neighbor at the starting corner (see Figure 2). We will prove in Section 2.2 that the limit 
exists $\omega$-a.s. and is nonrandom. It will turn out that $\psi_{A A}$ and $\psi_{B B}$ take on a simple form, whereas $\psi_{A B}$ and $\psi_{B A}$ do not.

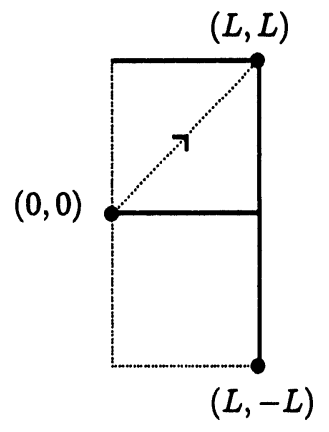

Fig. 2. Two neighboring blocks. The dashed line with arrow indicates that the coarsegrained path makes a step diagonally upwards.

Second, let $\mathscr{W}$ denote the class of all coarse-grained paths $\Pi=\left\{\Pi_{j}\right.$ : $j \in \mathbf{N}\}$ that step diagonally from corner to corner (see Figure 3, where each dashed line with arrow denotes a single step of $\Pi$ ). For $n \in \mathbf{N}, \Pi \in \mathscr{W}$, and $k, l \in\{A, B\}$, let

$\rho_{k l}^{\Omega}(\Pi, n)=\frac{1}{n} \sum_{j=1}^{n} 1\left\{\Pi_{j}\right.$ diagonally crosses a $k$-block in $\Omega$

that has an $l$-block in $\Omega$ appearing as

its neighbor at the starting corner\}.

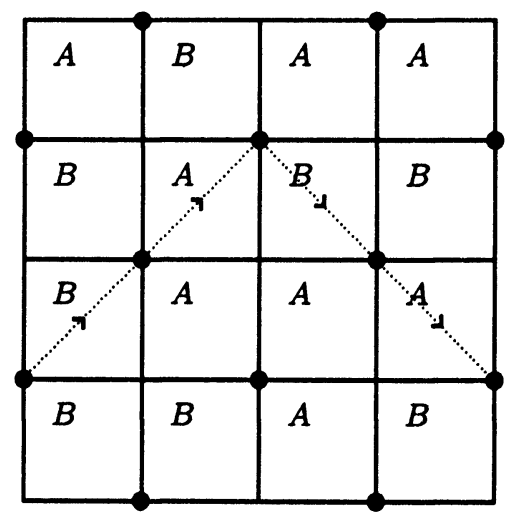

Fig. 3. $\Pi$ sampling $\Omega$. The dashed lines with arrows indicate the steps of $\Pi$.

Denote

$$
\rho^{\Omega}(\Pi, n)=\left(\rho_{k l}^{\Omega}(\Pi, n)\right)_{k, l \in\{A, B\}}
$$


which is a $2 \times 2$ matrix with nonnegative entries that sum up to 1 . Let $\mathscr{R}^{\Omega}(\Pi)$ denote the set of all limits points of the sequence $\left\{\rho^{\Omega}(\Pi, n): n \in \mathbf{N}\right\}$, and put

$$
\mathscr{R}^{\Omega}=\text { the closure of the set } \bigcup_{\Pi \in \mathscr{W}} \mathscr{R}^{\Omega}(\Pi) \text {. }
$$

Clearly, $\mathscr{R}^{\Omega}$ exists for all $\Omega$. Moreover, since $\Omega$ has a trivial sigma-field at infinity (i.e., all events not depending on finitely many coordinates of $\Omega$ have probability 0 or 1 ) and $\mathscr{R}^{\Omega}$ is measurable with respect to this sigma-field, we have

$$
\mathscr{R}^{\Omega}=\mathscr{R}(p) \quad \Omega \text {-a.s. }
$$

for some nonrandom closed set $\mathscr{R}(p)$. This set, which depends on the parameter $p$ controlling $\Omega$, will be analyzed in Section 3.2. It is the set of all possible limit points of the frequencies at which the four pairs of adjacent blocks can be seen along an infinite coarse-grained path.

Let $\mathscr{A}$ be the set of $2 \times 2$ matrices whose entries $\geqslant 2$. The starting point of our paper is the following representation of the free energy.

Theorem 1.3.1. (i) For all $(\alpha, \beta) \in \mathbf{R}^{2}$ and $p \in(0,1)$,

$$
\lim _{n \rightarrow \infty} f_{n, L_{n}}^{\omega, \Omega}=f=f(\alpha, \beta ; p)
$$

exists $\omega, \Omega$-a.s., is finite and nonrandom, and is given by

$$
f=\sup _{\left(a_{k l}\right) \in \mathscr{A}} \sup _{\left(\rho_{k l}\right) \in \mathscr{R}(p)} \frac{\sum_{k, l} \rho_{k l} a_{k l} \psi_{k l}\left(a_{k l}\right)}{\sum_{k, l} \rho_{k l} a_{k l}} .
$$

(ii) The function $(\alpha, \beta) \mapsto f(\alpha, \beta ; p)$ is convex on $\mathbf{R}^{2}$ for all $p \in(0,1)$.

(iii) The function $p \mapsto f(\alpha, \beta ; p)$ is continuous on $(0,1)$ for all $(\alpha, \beta) \in \mathbf{R}^{2}$.

(iv) For all $(\alpha, \beta) \in \mathbf{R}^{2}$ and $p \in(0,1)$,

$$
f(\alpha, \beta ; p)=f(\beta, \alpha ; 1-p), \quad f(\alpha, \beta ; p)=\frac{1}{2}(\alpha+\beta)+f(-\beta,-\alpha ; p) .
$$

Theorem 1.3.1(i), which will be proved in Section 3.1, says that the limiting free energy per step is self-averaging in both $\omega$ and $\Omega$, and equals the average of the limiting free energies per step associated with the four pairs of adjacent blocks, weighted and optimized according to the frequencies at which these four pairs are visited by the coarse-grained path and the fraction of time spent in each of them by the path. Assumption (1.2.3) is crucial, since it provides the separation of the path scale and the block scale, thereby separating the self-averaging in $\omega$ and $\Omega$. Theorem 1.3.1(ii), which will be proved in Section 3.1 also, is standard. Theorem 1.3.1(iii) is a consequence 
of the fact that $p \mapsto \mathscr{R}(p)$ is continuous in the Hausdorff metric, which will be proved in Section 3.2. Theorem 1.3.1(iv) is immediate from (1.2.1) upon interchanging the two monomer types and/or the two block types.

In view of Theorem 1.3.1(iv), we may without loss of generality restrict to the cone

$$
\mathrm{CONE}=\left\{(\alpha, \beta) \in \mathbf{R}^{2}: \alpha \geqslant|\beta|\right\} .
$$

The upper half of the cone is the physically most relevant part, but we will see that also the lower half of the cone is of interest. Note that $A A$-matches are favored over $B B$-matches. This will be crucial throughout the paper.

The behavior of $f$ as a function of $(\alpha, \beta)$ is different for $p \geqslant p_{c}$ and $p<p_{c}$, where $p_{c} \approx 0.64$ is the critical percolation density for directed bond percolation on the square lattice. The reason is that the coarse-grained paths $\Pi$, which determine the set $\mathscr{R}(p)$, sample $\Omega$ just like paths in directed bond percolation on the square lattice rotated by 45 degrees sample the percolation configuration (see Figure 3).

1.4. Supercritical case $p \geqslant p_{c}$. The entropy per step of the walk in a single block, subject to (1.2.3), is

$$
\kappa=\lim _{n \rightarrow \infty} \frac{1}{n} \ln \left|\mathscr{W}_{n, L_{n}}\right|
$$

In Section 2.1 we will see that $\kappa=\frac{1}{2} \ln 5$. This number is special to our model.

Our first theorem identifies the two phases, which turn out not to depend on $p$.

Theorem 1.4.1. Let $p \geqslant p_{c}$. Then $f(\alpha, \beta ; p)=f(\alpha, \beta)$, and $(\alpha, \beta) \mapsto$ $f(\alpha, \beta)$ is nonanalytic along the curve in CONE separating the two regions

$$
\begin{aligned}
& \mathscr{D}=\text { delocalized regime }=\left\{(\alpha, \beta) \in \mathrm{CONE}: f(\alpha, \beta)=\frac{1}{2} \alpha+\kappa\right\}, \\
& \mathscr{L}=\text { localized regime }=\left\{(\alpha, \beta) \in \mathrm{CONE}: f(\alpha, \beta)>\frac{1}{2} \alpha+\kappa\right\} .
\end{aligned}
$$

The intuition behind Theorem 1.4.1, which will be proved in Section 4.1.1, is as follows. The $A$-blocks (almost) percolate. Therefore the polymer has the option of moving to the (incipient) infinite cluster of $A$ blocks and staying in that infinite cluster forever, thus seeing only $A A$-blocks. In doing so, it loses an entropy of at most $o\left(n / L_{n}\right)=o(n)$, it gains an energy $\frac{1}{2} \alpha n+o(n)$ (because only half of its monomers are matched), and it gains an entropy $\kappa n+o(n)$. Alternatively, the path has the option of following the boundary of the infinite cluster, at least part of the time, during which it sees $A B$-blocks and (when $\beta \geqslant 0$ ) gains more energy by matching more than half of its monomers. Consequently, $f(\alpha, \beta) \geqslant \frac{1}{2} \alpha+\kappa$. The boundary 
between the two regimes in (1.4.2) corresponds to the crossover where one option takes over from the other.

Our second theorem gives an explicit classification of the two phases in terms of the free energies $\psi_{A A}$ and $\psi_{A B}$.

Theorem 1.4.2. Let $p \geqslant p_{c}$. Then

$$
\begin{aligned}
& \mathscr{D}=\left\{(\alpha, \beta) \in \mathrm{CONE}: S_{A B}=S_{A A}\right\}, \\
& \mathscr{L}=\left\{(\alpha, \beta) \in \mathrm{CONE}: S_{A B}>S_{A A}\right\},
\end{aligned}
$$

where

$$
S_{k l}=S_{k l}(\alpha, \beta)=\sup _{a \geqslant 2} \psi_{k l}(\alpha, \beta ; a) .
$$

We have $S_{A B} \geqslant S_{A A}$ for all $(\alpha, \beta)$, because in an $A B$-block the path may spend all of its time in the half that is $A$, in which case it is not aware of the presence of the half that is $B$ (see Figure 4). Theorem 1.4.2, which will be proved in Section 4.1.1 also, says that the critical curve marks those parameter values where $=$ changes to $>$.
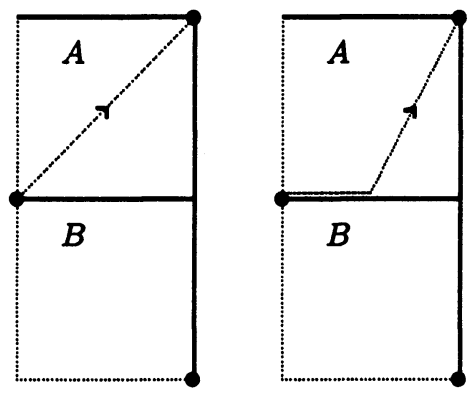

Fig. 4. Two possible strategies inside an $A B$-block: The path can either move straight across or move along the interface for awhile and then move across. Both strategies correspond to a coarse-grained step diagonally upwards.

Our third theorem gives the qualitative properties of the critical curve separating $\mathscr{D}$ and $\mathscr{L}$ (see Figure 5).

\section{Theorem 1.4.3. Let $p \geqslant p_{c}$.}

(i) For every $\alpha \geqslant 0$ there exists a $\beta_{c}(\alpha) \in[0, \alpha]$ such that the copolymer is delocalized if $-\alpha \leqslant \beta \leqslant \beta_{c}(\alpha)$, localized if $\beta_{c}(\alpha)<\beta \leqslant \alpha$.

(ii) The function $\alpha \mapsto \beta_{c}(\alpha)$ is continuous, nondecreasing, and concave on $[0, \infty)$.

(iii) There exists an $\alpha^{*} \in(0, \infty)$ such that $\beta_{c}(\alpha)=\alpha$ if $\alpha \leqslant \alpha^{*}$, $\beta_{c}(\alpha)<\alpha$ if $\alpha>\alpha^{*}$. Moreover,

$$
\lim _{\alpha \downarrow \alpha^{*}} \frac{\alpha-\beta_{c}(\alpha)}{\alpha-\alpha^{*}} \in[0,1)
$$


(iv) There exists a $\beta^{*} \in\left[\alpha^{*}, \infty\right)$ such that $\lim _{\alpha \rightarrow \infty} \beta_{c}(\alpha)=\beta^{*}$.

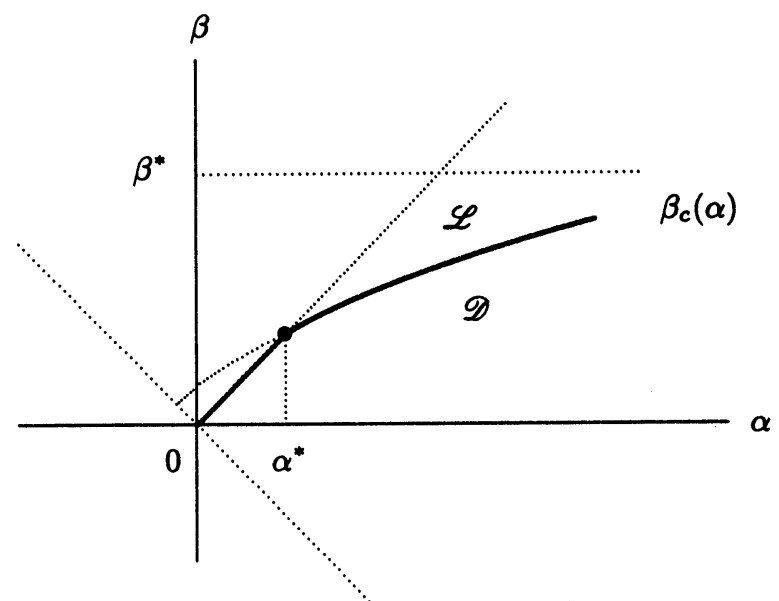

Fig. 5. Qualitative picture of $\alpha \mapsto \beta_{c}(\alpha)$ for $p \geqslant p_{c}$. The curved dotted line is the analytic continuation outside CONE of the part off the diagonal.

It is clear from (1.4.2) that the part off the diagonal is a critical line. We will see in Section 4.2.3 that also the part on the diagonal is a critical line. Theorem 1.4.3, which will be proved in Section 4.1.2, says that the critical curve follows the diagonal for $\alpha \in\left[0, \alpha^{*}\right]$, moves off the diagonal at $\alpha=\alpha^{*}$ with a slope discontinuity, and has a finite asymptote for large $\alpha$. The concavity of the curve implies that it is strictly increasing as long as it is below the asymptote. We are not able to exclude that the curve hits the asymptote, nor that it follows the diagonal all the way up to the asymptote, but we expect this not to happen. We will see in Section 4.1.2 that the curved dotted line crosses the vertical axis at $\left(0, \alpha_{0}\right)$ with $\alpha_{0} \approx 0.125$. We have no numerical values for $\alpha^{*}$ and $\beta^{*}$. We will show in Section 4.1.2 that $\beta^{*} \in[\ln 2,8 \ln 3)$. Clearly, $\alpha^{*} \in\left[\alpha_{0}, \beta^{*}\right]$.

To prove Theorem 1.4.3, we will reformulate the criterion $S_{A B}>S_{A A}$ in terms of a criterion for the free energy of a model with a single linear interface. This reformulation, which will be given in Section 2.3, is crucial in allowing us to get a handle on the critical curve in Figure 5.

We will see in Section 4.1.1 that $\mathscr{D}$ corresponds to the situation where the polymer is fully $A$-delocalized (i.e., it spends almost all of its time away from the interface deep inside the $A$-blocks), while $\mathscr{L}$ corresponds to the situation where the polymer is partially $A B$-delocalized (i.e., it spends a positive fraction of its time near those interfaces where it diagonally crosses the $A$-block rather than the $B$-block).

1.5. Subcritical case $p<p_{c}$. In the subcritical percolation regime, the analogue of the critical curve in Figure 5 turns out to depend on $p$ and to be much more difficult to characterize. We begin with some definitions. 
Let

$$
\rho^{*}(p)=\sup _{\left(\rho_{k l}\right) \in \mathscr{R}(p)}\left[\rho_{A A}+\rho_{A B}\right] .
$$

This is the maximal frequency of $A$-blocks crossed by an infinite coarsegrained path (recall (1.3.3)-(1.3.6)). The graph of $p \mapsto \rho^{*}(p)$ looks like:

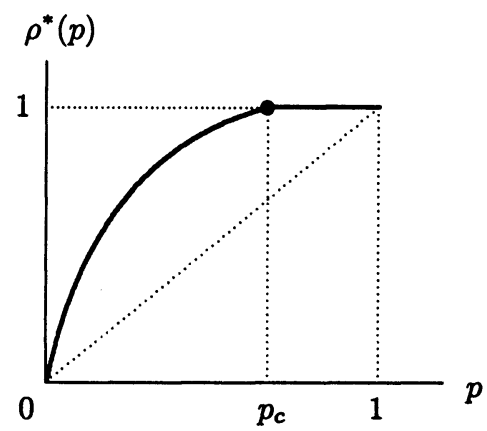

Fig. 6. Qualitative picture of $p \mapsto \rho^{*}(p)$.

Further details will be given in Section 3.2.

For $x, y \geqslant 2$, let $u(x)=u(\alpha ; x)$ and $v(y)=v(\beta ; y)$ be defined by

$$
\begin{aligned}
& x u(x)=\frac{1}{2} \alpha x+\ln 2+\frac{1}{2} x \ln x-\frac{1}{2}(x-2) \ln (x-2), \\
& y v(y)=\frac{1}{2} \beta y+\ln 2+\frac{1}{2} y \ln y-\frac{1}{2}(y-2) \ln (y-2) .
\end{aligned}
$$

For $\rho \in(0,1)$, let

$$
F(\rho)=F(\alpha, \beta ; \rho)=\sup _{x, y \geqslant 2} \frac{\rho x u(x)+(1-\rho) y v(y)}{\rho x+(1-\rho) y} .
$$

This variational formula will be analyzed in Section 2.5. There we will see that $(\alpha, \beta) \mapsto F(\alpha, \beta ; \rho)$ is analytic on $\mathbf{R}^{2}$ for all $\rho \in(0,1)$.

The following is the analogue of Theorem 1.4.1, and will be proved in Section 4.2.1.

Theorem 1.5.1. Let $p<p_{c}$. Then $(\alpha, \beta) \mapsto f(\alpha, \beta ; p)$ is nonanalytic along the curve in CONE separating the two regions

$$
\begin{aligned}
\mathscr{D} & =\text { delocalized regime } \\
& =\left\{(\alpha, \beta) \in \mathrm{CONE}: f(\alpha, \beta ; p)=F\left(\alpha, \beta ; \rho^{*}(p)\right)\right\} \\
\mathscr{L} & =\text { localized regime }=\left\{(\alpha, \beta) \in \mathrm{CONE}: f(\alpha, \beta ; p)>F\left(\alpha, \beta ; \rho^{*}(p)\right)\right\} .
\end{aligned}
$$


The intuition behind Theorem 1.5.1 is as follows. We will see in Section 2.2.1 that $\psi_{A A}(a)=u(a)$ and $\psi_{B B}(a)=v(a)$. In the delocalized regime, the polymer stays away from the $A B$-interface. For the free energy this means that no difference is felt between $\psi_{A B}, \psi_{A A}$ and between $\psi_{B A}, \psi_{B B}$. Therefore in this regime the variational formula in (1.3.8) effectively reduces to

$$
f=\sup _{\left(a_{k l}\right) \in \mathscr{A}} \sup _{\left(\rho_{k l}\right) \in \mathscr{R}(p)} \frac{\rho_{A} a_{A A} \psi_{A A}\left(a_{A A}\right)+\rho_{B} a_{B B} \psi_{B B}\left(a_{B B}\right)}{\rho_{A} a_{A A}+\rho_{B} a_{B B}},
$$

where $\rho_{A}=\rho_{A A}+\rho_{A B}$ and $\rho_{B}=\rho_{B A}+\rho_{B B}$ are the frequencies at which the polymer diagonally traverses $A$-blocks and $B$-blocks, while $a_{A A}$ and $a_{B B}$ are the respective times spent inside these blocks. The first supremum amounts to optimizing over $a_{A A}, a_{B B} \geqslant 2$. Since $A A$-matches are preferred over $B B$ matches, implying $\psi_{A A} \geqslant \psi_{B B}$, the second supremum is taken at the largest possible value of $\rho_{A}=1-\rho_{B}$ in $\mathscr{R}(p)$, which is $\rho^{*}(p)$. Hence, putting $a_{A A}=x$ and $a_{B B}=y$, we get $f=F\left(\alpha, \beta ; \rho^{*}(p)\right)$. In the localized regime, on the other hand, the polymer spends part of its time near $A B$-interfaces or $B A$-interfaces, in which case a difference is felt between $\psi_{A B}, \psi_{A A}$ and/or between $\psi_{B A}, \psi_{B B}$, and the free energy is larger. In Section 4.2 .1 we will make the above intuition rigorous.

Comparing the first lines of (1.4.2) and (1.5.4), we see that the free energy in the supercritical delocalized regime is a function of $\alpha$ only and has a simple linear form, whereas the free energy in the subcritical delocalized regime is a function of $\alpha, \beta, \rho^{*}(p)$ and has a form that is rather more complicated. For $\rho=1,(1.5 .2)$ and (1.5.3) yield $F(\alpha, \beta ; 1)=\sup _{x \geqslant 2} u(x)=u\left(\frac{5}{2}\right)=$ $\frac{1}{2} \alpha+\frac{1}{2} \ln 5$. This explains the connection between (1.4.2) and (1.5.4).

The following is the analogue of Theorem 1.4.2 and will be proved in Section 4.2.1.

Theorem 1.5.2. Let $p<p_{c}$. Then

$$
\begin{aligned}
& \mathscr{D}=\left\{(\alpha, \beta) \in \mathrm{CONE}: \psi_{A B}(\bar{x})=\psi_{A A}(\bar{x}) \text { and } \psi_{B A}(\bar{y})=\psi_{B B}(\bar{y})\right\} \\
& \mathscr{L}=\left\{(\alpha, \beta) \in \mathrm{CONE}: \psi_{A B}(\bar{x})>\psi_{A A}(\bar{x}) \text { or } \psi_{B A}(\bar{y})>\psi_{B B}(\bar{y})\right\}
\end{aligned}
$$

where $\bar{x}=\bar{x}\left(\alpha, \beta ; \rho^{*}(p)\right)$ and $\bar{y}=\bar{y}\left(\alpha, \beta ; \rho^{*}(p)\right)$ are the unique maximizers of $F\left(\alpha, \beta ; \rho^{*}(p)\right)$, i.e., of the variational formula in (1.5.3) for $\rho=\rho^{*}(p)$.

Theorem 1.5.2 says that the crossover into the localized regime occurs when the difference between $\psi_{A B}, \psi_{A A}$ or between $\psi_{B A}, \psi_{B B}$ is felt at the minimizers of the variational formula for the delocalized regime.

Comparing (1.4.3) and (1.5.6), we see that the crossover into the supercritical localization regime occurs when the maxima of $\psi_{A B}, \psi_{A A}$ separate, whereas the crossover into the subcritical localization regime occurs 
when $\psi_{A B}, \psi_{A A}$ or $\psi_{B A}, \psi_{B B}$ separate at specific locations, which themselves depend on $\alpha, \beta, \rho^{*}(p)$.

We will see in Section 4.2.2 that $\psi_{B A}(\bar{y})=\psi_{B B}(\bar{y})$ implies $\psi_{A B}(\bar{x})=$ $\psi_{A A}(\bar{x})$. Hence, (1.5.6) in fact reduces to

$$
\begin{aligned}
& \mathscr{D}=\left\{(\alpha, \beta) \in \mathrm{CONE}: \psi_{B A}(\bar{y})=\psi_{B B}(\bar{y})\right\}, \\
& \mathscr{L}=\left\{(\alpha, \beta) \in \mathrm{CONE}: \psi_{B A}(\bar{y})>\psi_{B B}(\bar{y})\right\}
\end{aligned}
$$

This is to be interpreted as saying that, when the critical curve is crossed from $\mathscr{D}$ to $\mathscr{L}$, localization occurs in the $B A$-blocks rather than in the $A B$-blocks. The intuitive explanation is as follows. In the delocalized phase the polymer spends positive fractions of its time in the $A$-blocks and in the $B$-blocks (the $A$-blocks do not percolate). Because $A A$-matches are preferred over $B B$-matches, there is a larger reward for the polymer to $B A$-localize (stay close to the interface when diagonally crossing a $B$-block) than to $A B$-localize (stay close to the interface when diagonally crossing an $A$ block).

The following is the analogue of Theorem 1.4.3, and will be proved in Section 4.2.2. Two constants $0<\alpha_{0}<\alpha_{1}<\infty$ appear, which will be identified in Section 2.2 .

Theorem 1.5.3. Let $p<p_{c}$.

(i) $\partial \mathscr{D}$ lies on or below the supercritical curve.

(ii) $\partial \mathscr{D}$ is continuous and intersects each line from the origin with slope in $[-1,1)$ at most once.

(iii) $\partial \mathscr{D}$ contains the diagonal segment $\left\{(\alpha, \alpha): \alpha \in\left[0, \alpha^{*}\right]\right\}$, with $\alpha^{*}$ the same constant as in Theorem 1.4.3 (iii), but lies below the diagonal elsewhere.

(iv) There exists an $\alpha^{*}(p) \in(0, \infty)$ such that the intersection of $\partial \mathscr{D}$ with the lower half of CONE is the linear segment $\left\{\left(\beta+\alpha^{*}(p), \beta\right)\right.$ : $\left.\beta \in\left[-\frac{1}{2} \alpha^{*}(p), 0\right]\right\}$.

(v) As $p \downarrow 0, \partial \mathscr{D}$ converges to the union of the diagonal segment in (iii) and the mirror image of the analytic continuation of the supercritical curve outside CONE (i.e., the mirror image of the curved dotted line in Figure 5). In particular, $\lim _{p \downarrow 0} \alpha^{*}(p)=\alpha_{0}$.

(vi) $A s p \uparrow p_{c}, \partial \mathscr{D}$ does not converge to the supercritical curve in Figure 5. In particular, $\lim _{p \uparrow p_{c}} \alpha^{*}(p)=\alpha_{1}$.

It is clear from (1.5.4) that the part off the diagonal is a critical line. We will see in Section 4.2.3 that also the part on the diagonal is a critical line.

We will see in Section 2.2 that $\mathscr{D}$ corresponds to the situation where the polymer is fully delocalized into the A-blocks and the B-blocks, while $\mathscr{L}$ corresponds to the situation where the polymer is partially $B A$-localized. We expect that $\mathscr{D}$ is strictly increasing in $p$ and that $\alpha^{*}>\alpha_{1}$, but we are unable to prove this. The curved dotted line crosses the horizontal axis at $\alpha_{0}$. 


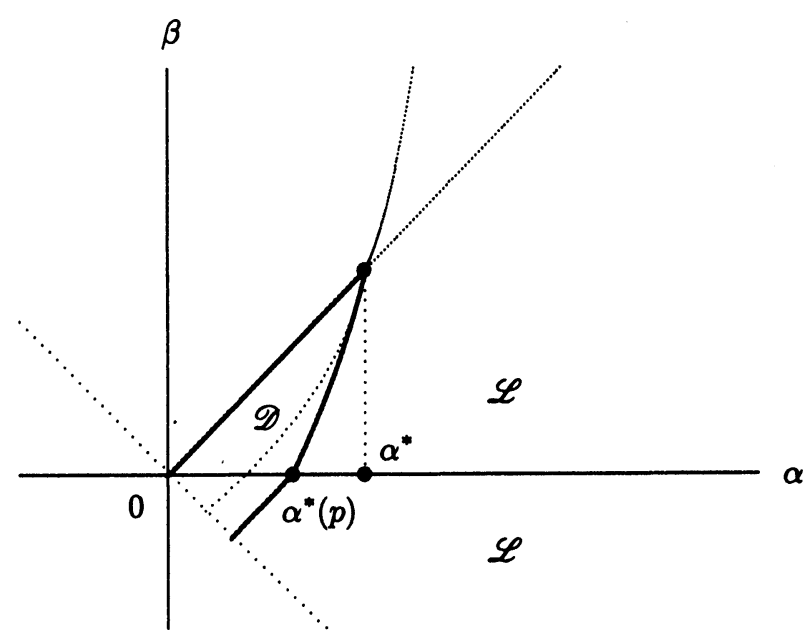

Fig. 7. Qualitative picture of $\partial \mathscr{D}$ for $p<p_{c}$. The curved dotted line is the mirror image of the union of the supercritical curve off the diagonal and its analytic continuation outside CONE (see Figure 5).

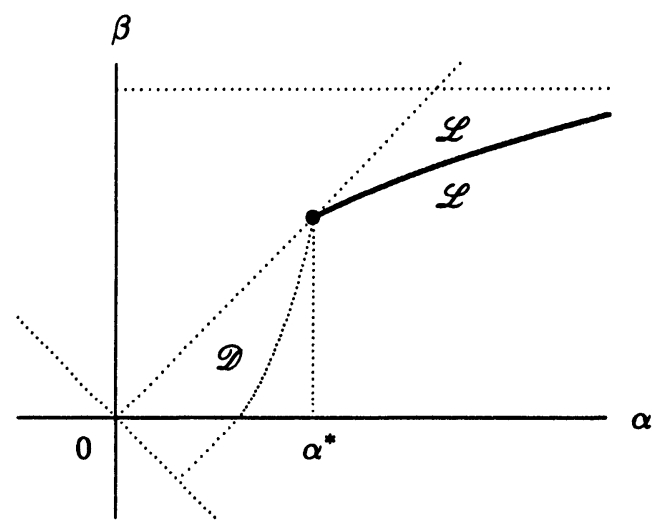

Fig. 8. Conjectured critical line inside $\mathscr{L}$ for $p<p_{c}$.

We will see in Section 4.3 that $\mathscr{L}$ contains a second curve (see Figure 8) at which a phase transition occurs from partially $B A$-localized to partially $B A$-localized and partially $A B$-localized. Qualitatively, this curve behaves like the supercritical curve (e.g., it also starts at the point $\left(\alpha^{*}, \alpha^{*}\right)$ ), but. unfortunately we know little about it. We expect it to be strictly increasing in $\alpha$. We expect it to move down as $p$ increases. We do know that it converges to the supercritical curve as $p \uparrow p_{c}$.

To prove Theorem 1.5.3, we will reformulate the criteria $\psi_{A B}(\bar{x})>$ $\psi_{A A}(\bar{x})$ and $\psi_{B A}(\bar{y})>\psi_{B B}(\bar{y})$ in terms of criteria for the free energy of a 
model with a single linear interface. This reformulation, which will be given in Section 2.4, is again crucial in allowing us to get a handle on the critical curve in Figure 7.

1.6. Heuristic explanation of the phase diagram. The physical background of the three critical curves in Figures 5, 7, and 8 is as follows.

(a) $p \geqslant p_{c}$ : Consider the boundary $\partial \mathscr{D}$ sketched in Figure 5 . Choose a point $(\widehat{\alpha}, \widehat{\beta})$ inside $\mathscr{D}$. Then, since $p \geqslant p_{c}$ and $\alpha \geqslant \beta$, the polymer spends almost all of its time deep inside $A$-blocks. Now increase $\beta$ but keep $\alpha=\widehat{\alpha}$ fixed. Then there will be a larger energetic advantage for the polymer to move some of its monomers from the $A$-blocks to the $B$-blocks by crossing the interface inside the $A B$-block pairs. There is some entropy loss associated with doing so. The polymer has three options: (i) it may place all $A$-monomers in the $A$-blocks and no monomers in the $B$-blocks (resulting in all energy coming from $A A$-matches and some entropy); (ii) it may place all $A$-monomers in the $A$-blocks and a positive fraction of $B$-monomers in the $B$-blocks (resulting in a higher energy and a lower entropy); (iii) it may sacrifice some fraction of $A A$-matches to get a larger fraction of $B B$-matches (resulting in an even higher energy and an even lower entropy). If $\beta$ is large enough, then the energy advantage will dominate, so that $A B$-localization sets in. The value at which this happens depends on $\widehat{\alpha}$ and is strictly positive. Since the entropy loss is finite, for $\widehat{\alpha}$ large enough the energy-entropy competition plays out not only below the diagonal, but also below a horizontal asymptote. The larger the value of $\hat{\alpha}$, the larger the value of $\beta$, where $A B$-localization sets in. This explains why the part of $\partial \mathscr{D}$ off the diagonal moves to the right and up.

(b) $p<p_{c}$ : First consider the boundary $\partial \mathscr{D}$ sketched in Figure 7. Choose a point $(\widehat{\alpha}, \widehat{\beta})$ inside $\mathscr{D}$. Since $p<p_{c}$, the polymer spends almost all of its time deep inside $A$-blocks and $B$-blocks. Now increase $\alpha$ but keep $\beta=\widehat{\beta}$ fixed. Then, while remaining delocalized, the polymer will spend more time in the $A$-blocks and less time in the $B$-blocks, trying to lower its energy with some attendant loss of entropy. As $\alpha$ increases further, there will be a larger energetic advantage for the polymer to move some of its monomers from the $B$-blocks to the $A$-blocks by crossing the interface inside the $B A$-block pairs. If $\alpha$ is large enough, then the energetic advantage will dominate, so that $B A$-localization sets in eventually. The value of $\alpha$ at which this happens depends on $\widehat{\beta}$. A larger value of $\widehat{\beta}$ means that the polymer spends more time in the $B$-blocks (at fixed $\alpha$ ) with larger entropy. Consequently, more entropy will be lost on $B A$-localization and the value of $\alpha$, where $B A$-localization sets in will be larger. This explains why the part of $\partial \mathscr{D}$ off the diagonal moves to the right and up. Similarly, if $p$ decreases, then the polymer hits more $B$-blocks, and to compensate for the loss of energy it will spend more time in an $A$-block when it hits one and less time in a $B$-block when it hits one (at fixed $\widehat{\alpha}$ and $\widehat{\beta}$ ). Consequently, 
less entropy will be lost on $B A$-localization and the value of $\alpha$ (at fixed $\widehat{\beta}$ ) where $B A$-localization sets in will be smaller. This explains why $\mathscr{D}$ shrinks with $p$.

If $\beta \leqslant 0$, then there is a penalty for having $B$-monomers in $B$-blocks. Therefore, when the polymer $B A$-localizes, it will spend all the time it runs along the interface in the $A$-block and then shoot through the interface to spend its remaining time in the $B$-block (on its way to the diagonally opposite corner). Hence, the energy-entropy competition only depends on the difference $\alpha-\beta$. This explains why there is a degeneration of the critical curve into a linear segment.

In the limit as $p \downarrow 0$, the density of $A$-blocks tends to zero and so the polymer spends more and more of its time in $B$-blocks. Therefore the localization mechanism looks more and more like that for the supercritical curve with $\alpha \rightarrow \beta$ and $p \uparrow 1$.

Next consider the curve separating $\mathscr{L}$ sketched in Figure 8 . Choose a point $(\widehat{\alpha}, \widehat{\beta})$ inside $\mathscr{L}$. Now increase $\beta$ but keep $\alpha=\widehat{\alpha}$ fixed. Then, as before, an energy-entropy competition sets in. The polymer has the same three options inside $A B$-blocks as in the supercritical case, and therefore the curve has the same qualitative behavior. In the limit as $p \uparrow p_{c}$, the polymer spends more and more of its time in $A$-blocks. Therefore the $A B$-localization mechanism looks more and more like that for the supercritical curve. If $p$ decreases, then the polymer hits more $B$-blocks, and to compensate for the loss of energy it will spend more time in an $A$-block when it hits one and less time in a $B$-block when it hits one (at fixed $\widehat{\alpha}$ and $\widehat{\beta}$ ). Consequently, more entropy will be lost on $A B$-localization and the value of $\beta$ (at fixed $\widehat{\alpha}$ ) where $A B$-localization sets in will be larger. This explains why the curve moves up as $p$ decreases.

In Section 4.2.3 the full phase diagram is drawn, i.e., its extension from CONE to $\mathbf{R}^{2}$ with the help of the two symmetry properties stated in (1.3.9).

1.7. Open problems. The fine details of the two subcritical curves remain to be settled. Here are some further open problems:

1. Are the critical curves smooth off the diagonal and inside the first quadrant? Even for the model with a single linear interface this question has not been settled.

2. For $p \geqslant p_{c}$, is the free energy infinitely differentiable inside the localized phase? For the model with a single linear interface this was proved by Giacomin and Toninelli [13]. Is the same true for $p<p_{c}$ in the interior of the two subphases of the localized phase?

3. Our phase transitions are defined in terms of a nonanalyticity in the free energy. Heuristically, they correspond to the path changing its behavior from being fully delocalized away from the interfaces to being partially localized near the interfaces (in the subcritical case even in two possible ways). How can we prove that the path actually has this behavior under the 
transformed path measure $P_{n, L_{n}}^{\omega, \Omega}(\pi)=\left(Z_{n, L_{n}}^{\omega, \Omega}\right)^{-1} \exp \left[-H_{n, L_{n}}^{\omega, \Omega}(\pi)\right]$ for large $n$ ? For the model with a single linear interface this question was settled in [2] and [11].

4. How does the free energy behave near the critical curve? For the model with a single linear interface it was shown by Giacomin and Toninelli [12] that the phase transition is at least of second order. $\mathrm{Nu}-$ merical results in [7] suggest that the same is true for the self-avoiding walk model.

5. The coarse-graining expressed by (1.2.3) and the restriction that the polymer can enter and exit a pair of neighboring blocks only at diagonally opposite corners are necessary to make the model mathematically tractable. Indeed, the corner restriction and $L_{n} \rightarrow \infty$ guarantee that the polymer «sees one pair of blocks at a time and self-averages in $\omega$ in each block, which is why the free energy can be decomposed into contributions coming from single pairs of blocks, while $L_{n} / n \rightarrow 0$ guarantees that the polymer «sees many blocks» and self-averages in $\Omega$, which is why percolation effects enter. What happens when we remove the corner restriction? What happens when the blocks have random sizes?

\section{Preparations}

In Section 2.1 we compute entropies for paths that cross a block and paths that run along an interface. In Section 2.2 we derive a formula for $\psi_{k l}$ in (1.3.2) and $S_{k l}$ in (1.4.4). In Section 2.3 we deduce a criterion for localization when $p \geqslant p_{c}$ in terms of the free energy for the model with a single linear interface. In Section 2.4 we do the same when $p<p_{c}$. In Section 2.5 we analyse the supremum $\rho^{*}(p)$ in (1.5.1) and the variational formula for $F(\rho)$ in (1.5.3).

2.1. Path entropies. The results in this section are based on straightforward computations, but are crucial for the rest of the paper.

2.1.1. Paths crossing a block. An important ingredient in the identification of $\psi_{k l}(a), k, l \in\{A, B\}$, is the following combinatorial lemma. Let $\mathrm{DOM}=\{(a, b): a \geqslant 1+b, b \geqslant 0\}$. For $(a, b) \in \mathrm{DOM}$, let $N_{L}(a, b)$ denote the number of $a L$-step self-avoiding directed paths from $(0,0)$ to $(b L, L)$ whose vertical displacement stays within $(-L, L]$ ( $a L$ and $b L$ are integer). Let

$$
\kappa(a, b)=\lim _{L \rightarrow \infty} \frac{1}{a L} \ln N_{L}(a, b) .
$$

Lemma 2.1.1. (i) $\kappa(a, b)$ exists and is finite for all $(a, b) \in \mathrm{DOM}$.

(ii) $(a, b) \mapsto a \kappa(a, b)$ is continuous and strictly concave on DOM and analytic on the interior of DOM.

(iii) For all $a \geqslant 2$,

$$
a \kappa(a, 1)=\ln 2+\frac{1}{2}[a \ln a-(a-2) \ln (a-2)] .
$$


(iv) $\sup _{a \geqslant 2} \kappa(a, 1)=\kappa\left(a^{*}, 1\right)=\frac{1}{2} \ln 5$ with unique maximizer $a^{*}=\frac{5}{2}$.

(v) $\left(\frac{\partial}{\partial a} \kappa\right)\left(a^{*}, 1\right)=0$ and $a^{*}\left(\frac{\partial}{\partial b} \kappa\right)\left(a^{*}, 1\right)=\frac{1}{2} \ln \frac{9}{5}$.

$\mathrm{Pr}$ o of. (i) First we do the computation without the restriction on the vertical displacement. Later we show that putting in the restriction is harmless.

Let $N_{L}^{0}(a, b)$ denote the number of $a L$-step self-avoiding directed paths from $(0,0)$ to $(b L, L)$. Since such paths make $b L$ steps to the right, $\frac{1}{2}(a+$ $1-b) L$ steps upwards and $\frac{1}{2}(a-1-b) L$ steps downwards, we have

$$
\begin{aligned}
N_{L}^{0}(a, b)= & \sum_{k=1}^{b L}\left(\begin{array}{c}
b L \\
k
\end{array}\right)\left(\begin{array}{c}
\frac{1}{2}(a+1-b) L-1 \\
k-1
\end{array}\right) \\
& \times \sum_{l=1}^{b L-k}\left(\begin{array}{c}
b L-k \\
l
\end{array}\right)\left(\begin{array}{c}
\frac{1}{2}(a-1-b) L-1 \\
l-1
\end{array}\right) .
\end{aligned}
$$

Here, $k$ counts the number of columns, where the path moves upward, $l$ counts the number of columns, where the path moves downward, the first and the third binomial coefficient count the number of choices for these columns, while the second and the fourth binomial coefficient count the number of ways in which the prescribed number of steps can be distributed over these columns. Since, by Stirling's formula,

$$
\lim _{L \rightarrow \infty} \frac{1}{L} \ln \left(\begin{array}{l}
u L \\
v L
\end{array}\right)=u \ln u-v \ln v-(u-v) \ln (u-v), \quad 0 \leqslant v \leqslant u,
$$

we get, by putting $k=\delta L, l=\varepsilon L$, that

$$
a \kappa^{0}(a, b)=\lim _{L \rightarrow \infty} \frac{1}{L} \ln N_{L}^{0}(a, b)=\sup _{\delta, \varepsilon} f_{a b}(\delta, \varepsilon)
$$

with

$$
\begin{aligned}
f_{a b}(\delta, \varepsilon)= & b \ln b-2 \delta \ln \delta+\left(\frac{a+1-b}{2}\right) \ln \left(\frac{a+1-b}{2}\right) \\
& -\left(\frac{a+1-b}{2}-\delta\right) \ln \left(\frac{a+1-b}{2}-\delta\right)-2 \varepsilon \ln \varepsilon \\
& -(b-\delta-\varepsilon) \ln (b-\delta-\varepsilon)+\left(\frac{a-1-b}{2}\right) \ln \left(\frac{a-1-b}{2}\right) \\
& -\left(\frac{a-1-b}{2}-\varepsilon\right) \ln \left(\frac{a-1-b}{2}-\varepsilon\right) .
\end{aligned}
$$

Computing

$$
\begin{aligned}
& \frac{\partial f_{a b}}{\partial \delta}=\ln \left[\frac{\left(\frac{1}{2}(a+1-b)-\delta\right)(b-\delta-\varepsilon)}{\delta^{2}}\right], \\
& \frac{\partial f_{a b}}{\partial \varepsilon}=\ln \left[\frac{\left(\frac{1}{2}(a-1-b)-\varepsilon\right)(b-\delta-\varepsilon)}{\varepsilon^{2}}\right],
\end{aligned}
$$


and setting these derivatives equal to zero, we find that the maximizers $\delta_{a b}$ and $\varepsilon_{a b}$ of the right-hand side of (2.1.3) are solutions of quadratic equations, namely,

$$
\begin{aligned}
& 0=(1+b) \delta^{2}-(a+1) b \delta+\frac{a+1-b}{2} b^{2}, \\
& 0=(1-b) \varepsilon^{2}+(a-1) b \varepsilon-\frac{a-1-b}{2} b^{2},
\end{aligned}
$$

which leads to

$$
\begin{aligned}
& \delta_{a b}=\frac{b}{2(1+b)}\left[(a+1)-\sqrt{(a-b)^{2}+\left(b^{2}-1\right)}\right], \\
& \varepsilon_{a b}=\frac{b}{2(1-b)}\left[-(a-1)+\sqrt{(a-b)^{2}+\left(b^{2}-1\right)}\right],
\end{aligned}
$$

for $b \neq 1$, and

$$
\delta_{a 1}=\frac{1}{2}, \quad \varepsilon_{a 1}=\frac{a-2}{2(a-1)},
$$

for $b=1$. Substitution of (2.1.5), (2.1.6) into (2.1.3), (2.1.4) yields a formula for $a \kappa^{0}(a, b)$ in closed form. From this formula it is obvious that $(a, b) \mapsto$ $\kappa^{0}(a, b)$ is continuous on DOM and analytic on the interior of DOM.

It remains to show that the restriction on the vertical displacement has no effect in the limit as $L \rightarrow \infty$. This can be done by appealing to the reflection principle. Indeed, let $N_{L}^{\downarrow}(a, b)$ be the number of paths where the restriction of not moving above the line of height $L$ is inserted. Then $N_{L}^{\downarrow}(a, b)$ is the difference of two terms of the type $N_{L}^{0}(a, b)$ in (2.1.2), one with the path ending at $(b L, L+2)$ and one with the path ending at $(b L, L)$. A little computation shows that this difference equals $N_{L}^{0}(a, b)$ divided by a term that is growing at most polynomially fast in $L$. This polynomial factor does not affect the exponential asymptotics. A similar argument shows that the restriction of not moving below the line of height $-L+1$ is harmless as well. Hence

$$
\kappa(a, b)=\kappa^{0}(a, b) .
$$

(ii) Choose any $b_{1}, b_{2} \geqslant 0$ and $a_{1} \geqslant 1+b_{1}, a_{2} \geqslant 1+b_{2}$. Consider a block of height $L$ and width $\frac{1}{2}\left(b_{1}+b_{2}\right) L$, and partition this block into four parts by cutting it at height $\frac{1}{2} L$ and width $\frac{1}{2} b_{1} L$. The number of paths that cross the large block in $\frac{1}{2}\left(a_{1}+a_{2}\right) L$ steps is larger than or equal to the number of paths that cross the lower left block in $\frac{1}{2} a_{1} L$ steps times the number of paths that cross the upper right block in $\frac{1}{2} a_{2} L$ steps, i.e.,

$$
N_{L}^{0}\left(\frac{a_{1}+a_{2}}{2}, \frac{b_{1}+b_{2}}{2}\right) \geqslant N_{L / 2}^{0}\left(a_{1}, b_{1}\right) N_{L / 2}^{0}\left(a_{2}, b_{2}\right) .
$$


By (2.1.3) and (2.1.7), this proves that

$$
\frac{a_{1}+a_{2}}{2} \kappa\left(\frac{a_{1}+a_{2}}{2}, \frac{b_{1}+b_{2}}{2}\right) \geqslant \frac{1}{2} a_{1} \kappa\left(a_{1}, b_{1}\right)+\frac{1}{2} a_{2} \kappa\left(a_{2}, b_{2}\right),
$$

which is the concavity desired. Strict concavity follows from analyticity on the interior of DOM, because $a \kappa(a, b)$ clearly is not linear in either $a$ or $b$.

(iii) Substitute (2.1.6) into (2.1.3), (2.1.4) to get the formula for $a \kappa(a, 1)$ stated in (2.1.1).

(iv) Since

$$
\frac{d}{d a} \kappa(a, 1)=-a^{-2} \ln [2(a-2)],
$$

the supremum is uniquely attained at $a^{*}=\frac{5}{2}$, giving the claim.

(v) Compute, from (2.1.4),

$$
\begin{aligned}
\left(\frac{\partial}{\partial a} \kappa\right)(a, b)= & \left(\frac{\partial}{\partial a}\left(\frac{1}{a} f_{a b}\right)\right)\left(\delta_{a b}, \varepsilon_{a b}\right) \\
= & -\frac{1}{a^{2}} f_{a b}\left(\delta_{a b}, \varepsilon_{a b}\right)+\frac{1}{a}\left(\frac{\partial}{\partial a} f_{a b}\right)\left(\delta_{a b}, \varepsilon_{a b}\right)=-\frac{1}{a} \kappa(a, b) \\
& +\frac{1}{a} \frac{1}{2} \ln \left[\frac{\frac{1}{2}(a+1-b) \frac{1}{2}(a-1-b)}{\left(\frac{1}{2}(a+1-b)-\delta_{a b}\right)\left(\frac{1}{2}(a-1-b)-\varepsilon_{a b}\right)}\right]
\end{aligned}
$$

and

$$
\begin{aligned}
& \left(\frac{\partial}{\partial b} \kappa\right)(a, b)=\left(\frac{\partial}{\partial b}\left(\frac{1}{a} f_{a b}\right)\right)\left(\delta_{a b}, \varepsilon_{a b}\right)=\frac{1}{a}\left(\frac{\partial}{\partial b} f_{a b}\right)\left(\delta_{a b}, \varepsilon_{a b}\right) \\
& \quad=\frac{1}{2} \frac{1}{a} \ln \left[\frac{b^{2}\left(\frac{1}{2}(a+1-b)-\delta_{a b}\right)\left(\frac{1}{2}(a-1-b)-\varepsilon_{a b}\right)}{\left(b-\delta_{a b}-\varepsilon_{a b}\right)^{2} \frac{1}{2}(a+1-b) \frac{1}{2}(a-1-b)}\right]
\end{aligned}
$$

Setting $a=a^{*}=\frac{5}{2}, b=1, \delta_{a b}=\delta_{a^{*} 1}=\frac{1}{2}$ and $\varepsilon_{a b}=\varepsilon_{a^{* 1}}=\frac{1}{6}$, we get the claim.

2.1.2. Paths running along an interface. We also need the following analogue of Lemma 2.1.1. For $\mu \geqslant 1$, let $\widehat{N}_{L}(\mu)$ denote the number of $\mu L$-step self-avoiding paths from $(0,0)$ to $(L, 0)$ with no restriction on the vertical displacement ( $\mu L$ is integer). Let

$$
\widehat{\kappa}(\mu)=\lim _{L \rightarrow \infty} \frac{1}{\mu L} \ln \widehat{N}_{L}(\mu) .
$$

Lemma 2.1.2. (i) $\widehat{\kappa}(\mu)$ exists and is finite for all $\mu \geqslant 1$.

(ii) $\mu \mapsto \mu \widehat{\kappa}(\mu)$ is continuous and strictly concave on $[1, \infty)$ and analytic on $(1, \infty)$.

(iii) $\widehat{\kappa}(1)=0$ and $\mu \widehat{\kappa}(\mu) \sim \ln \mu$ as $\mu \rightarrow \infty$.

(iv) $\sup _{\mu \geqslant 1} \mu\left[\widehat{\kappa}(\mu)-\frac{1}{2} \ln 5\right]<\frac{1}{2} \ln \frac{9}{5}$. 
P r o o f. (i) Similarly as in (2.1.2),

$$
\widehat{N}_{L}(\mu)=\sum_{k=1}^{L}\left(\begin{array}{l}
L \\
k
\end{array}\right)\left(\begin{array}{c}
\frac{1}{2}(\mu-1) L-1 \\
k-1
\end{array}\right) \sum_{l=1}^{L-k}\left(\begin{array}{c}
L-k \\
l
\end{array}\right)\left(\begin{array}{c}
\frac{1}{2}(\mu-1) L-1 \\
l-1
\end{array}\right) .
$$

Again putting $k=\delta L, l=\varepsilon L$, we get

$$
\mu \widehat{\kappa}(\mu)=\lim _{L \rightarrow \infty} \frac{1}{L} \ln \widehat{N}_{L}(\mu)=\sup _{\delta, \varepsilon} f_{\mu}(\delta, \varepsilon)
$$

with

$$
\begin{aligned}
f_{\mu}(\delta, \varepsilon)= & -2 \delta \ln \delta-2 \varepsilon \ln \varepsilon-(1-\delta-\varepsilon) \ln (1-\delta-\varepsilon) \\
& -\left(\frac{\mu-1}{2}-\delta\right) \ln \left(\frac{\mu-1}{2}-\delta\right)-\left(\frac{\mu-1}{2}-\varepsilon\right) \\
& \times \ln \left(\frac{\mu-1}{2}-\varepsilon\right)+(\mu-1) \ln \left(\frac{\mu-1}{2}\right) .
\end{aligned}
$$

Computing

$$
\begin{aligned}
& \frac{\partial f_{\mu}}{\partial \delta}=\ln \left[\frac{\left(\frac{1}{2}(\mu-1)-\delta\right)(1-\delta-\varepsilon)}{\delta^{2}}\right] \\
& \frac{\partial f_{\mu}}{\partial \varepsilon}=\ln \left[\frac{\left(\frac{1}{2}(\mu-1)-\varepsilon\right)(1-\delta-\varepsilon)}{\varepsilon^{2}}\right]
\end{aligned}
$$

and setting these derivatives equal to zero, we find that the maximizers $\delta_{\mu}$ and $\varepsilon_{\mu}$ of the right-hand side of (2.1.11) are equal, $\delta_{\mu}=\varepsilon_{\mu}$, with $\delta_{\mu}$ the solution of the quadratic equation $0=\delta^{2}-\mu \delta+(\mu-1) / 2$, which leads to

$$
\delta_{\mu}=\frac{1}{2}\left[\mu-\sqrt{(\mu-1)^{2}+1}\right] .
$$

Substitution of (2.1.13) into (2.1.12) yields a formula for $\mu \widehat{\kappa}(\mu)$ in closed form. From this formula it is obvious that $\mu \mapsto \widehat{\kappa}(\mu)$ is continuous on $[1, \infty)$ and analytic on $(1, \infty)$.

(ii) Choose any $\mu_{1}, \mu_{2} \geqslant 1$. The number of $\frac{1}{2}\left(\mu_{1}+\mu_{2}\right) L$-step paths from $(0,0)$ to $(L, 0)$ is larger than or equal to the number of $\frac{1}{2} \mu_{1} L$-step paths from $(0,0)$ to $\left(\frac{1}{2} L, 0\right)$ times the number of $\frac{1}{2} \mu_{2} L$-step paths from $\left(\frac{1}{2} L, 0\right)$ to $(L, 0)$, i.e.,

$$
\widehat{N}_{L}\left(\frac{\mu_{1}+\mu_{2}}{2}\right) \geqslant \widehat{N}_{L / 2}\left(\mu_{1}\right) \widehat{N}_{L / 2}\left(\mu_{2}\right) \text {. }
$$

Via (2.1.10), this proves that

$$
\frac{\mu_{1}+\mu_{2}}{2} \widehat{\kappa}\left(\frac{\mu_{1}+\mu_{2}}{2}\right) \geqslant \frac{1}{2} \mu_{1} \widehat{\kappa}\left(\mu_{1}\right)+\frac{1}{2} \mu_{2} \widehat{\kappa}\left(\mu_{2}\right)
$$

which is the concavity desired. Strict concavity follows from smoothness on $(1, \infty)$, because $\mu \kappa(\mu)$ clearly is not linear in $\mu$. 
(iii) From (2.1.13) we see that $\delta_{1}\left(=\varepsilon_{1}\right)=0$. Hence (2.1.11), (2.1.12) give $\widehat{\kappa}(1)=0$. Similarly, if $\mu \rightarrow \infty$, then $\delta_{\mu}=\frac{1}{2}\left[1-1 /(2 \mu)+O\left(1 / \mu^{2}\right)\right]$ and hence $\mu \widehat{\kappa}(\mu) \sim \ln \mu$.

(iv) For any $a \geqslant 2,0<b \leqslant 1, \mu \geqslant 1$ such that $(\mu-1) b \leqslant a-2$, we have

$$
a \kappa(a, 1) \geqslant b \mu \widehat{\kappa}(\mu)+(a-b \mu) \kappa(a-b \mu, 1-b) .
$$

Indeed, any $a L$-step self-avoiding path from $(0,0)$ to $(L, L)$ may follow the interface over a distance $b L$ during $b \mu L$ steps and then wander away from the interface to the diagonally opposite corner over a distance $(1-b) L$ during $(a-b \mu) L$ steps (see Figure 4). Rewrite (2.1.14) as

$$
\mu \widehat{\kappa}(\mu) \leqslant \frac{1}{b}[a \kappa(a, 1)-(a-b \mu) \kappa(a-b \mu, 1-b)] .
$$

Choose $a=a^{*}$ and let $b \downarrow 0$, to obtain

$$
\mu \widehat{\kappa}(\mu) \leqslant \mu\left(\frac{\partial}{\partial a}(a \kappa)\right)\left(a^{*}, 1\right)+\left(\frac{\partial}{\partial b}(a \kappa)\right)\left(a^{*}, 1\right) .
$$

By Lemma 2.1.1(iv), (v), the right-hand side equals $\mu \frac{1}{2} \ln 5+\frac{1}{2} \ln \frac{9}{5}$. Since $\mu \geqslant 1$ is arbitrary, this proves that $\sup _{\mu \geqslant 1} \mu\left[\widehat{\kappa}(\mu)-\frac{1}{2} \ln 5\right] \leqslant \frac{1}{2} \ln \frac{9}{5}$, which is the claim with $\leqslant$ instead of $<$. A calculation with MAPLE gives that the supremum in the left-hand side is attained at $\mu \approx 2.12$ and equals $\approx 0.16$. The right-hand side equals 0.29 .

In Section 4.1 we will need two special values of $\alpha$, namely, $\alpha_{0}$ and $\alpha_{1}$ given by

$$
\begin{aligned}
& \sup _{\mu \geqslant 1} \mu\left[\widehat{\kappa}(\mu)+\frac{1}{2} \alpha_{0}-\frac{1}{2} \ln 5\right]=\frac{1}{2} \ln \frac{9}{5} \\
& \sup _{\mu \geqslant 1} \mu\left[\widehat{\kappa}(\mu)-\frac{1}{2} \ln 5\right]=\frac{1}{2} \ln \left[\frac{4 e^{-\alpha_{1}}\left(5+e^{-\alpha_{1}}\right)^{2}}{5\left(5-e^{-\alpha_{1}}\right)^{2}}\right] .
\end{aligned}
$$

It follows from Lemma 2.1.2(iii), (iv) that $\alpha_{0}, \alpha_{1}>0$. A calculation with MAPLE gives the values $\alpha_{0} \approx 0.125, \alpha_{1} \approx 0.154$.

2.2. Free energies per pair of blocks. In this section we identify $S_{k l}=S_{k l}(\alpha, \beta)$.

\subsubsection{Identification of $S_{A A}$ and $S_{B B}$.}

Proposition 2.2.1. For all $(\alpha, \beta) \in \mathbf{R}^{2}$,

$$
\begin{aligned}
S_{A A} & =\sup _{a \geqslant 2} \psi_{A A}(a)=\frac{1}{2} \alpha+\frac{1}{2} \ln 5, \\
S_{B B} & =\sup _{a \geqslant 2} \psi_{B B}(a)=\frac{1}{2} \beta+\frac{1}{2} \ln 5 .
\end{aligned}
$$

P r o o f. Recall (1.2.1) and (1.3.1), (1.3.2). For any $a L$-step path in an $A A$-block, about half of the monomers contribute $\alpha$ to the energy, because 
$\sum_{i=1}^{a L} 1\left\{\omega_{i}=A\right\}=\frac{1}{2} a L[1+o(1)] \omega$-a.s. as $L \rightarrow \infty$, while the remaining monomers contribute 0 to the energy. Hence

$$
\psi_{A A}(a)=\frac{1}{2} \alpha+\kappa(a, 1) .
$$

Now use Lemma 2.1.1(iv) to get the claim for $S_{A A}$. The proof for $S_{B B}$ is the same.

2.2.2. Identification of $S_{A B}$ and $S_{B A}$. It is harder to obtain information on $S_{A B}=\sup _{a \geqslant 2} \psi_{A B}(a)$ and $S_{B A}=\sup _{a \geqslant 2} \psi_{B A}(a)$, because these embody the effect of the presence of the $A B$-interface. We first consider the free energy per step when the path moves in the vicinity of a single linear interface $\mathscr{I}$ separating a liquid $A$ in the upper halfplane from a liquid $B$ in the lower halfplane including the interface itself. To that end, for $a \geqslant b>0$, let $\mathscr{W}_{a L, b L}$ denote the set of $a L$-step directed self-avoiding paths starting at $(0,0)$ and ending at $(b L, 0)$. Define

$$
\psi_{L}^{\omega, \mathscr{I}}(a, b)=\frac{1}{a L} \ln Z_{a L, b L}^{\omega, \mathscr{I}}
$$

with

$$
\begin{aligned}
Z_{a L, b L}^{\omega, \mathscr{I}} & =\sum_{\pi \in \mathscr{W}_{a L, b L}} \exp \left[-H_{a L}^{\omega, \mathscr{I}}(\pi)\right], \\
H_{a L}^{\omega, \mathscr{I}}(\pi) & =-\sum_{i=1}^{a L}\left(\alpha 1\left\{\omega_{i}=A, \pi_{i}>0\right\}+\beta 1\left\{\omega_{i}=B, \pi_{i} \leqslant 0\right\}\right),
\end{aligned}
$$

where $\pi_{i}>0$ means that the $i$-th step lies in the upper halfplane and $\pi_{i} \leqslant 0$ means that the $i$-th step lies in the lower halfplane or in the interface.

Lemma 2.2.1. For all $(\alpha, \beta) \in \mathbf{R}^{2}$ and $a \geqslant b>0$,

$$
\lim _{L \rightarrow \infty} \psi_{L}^{\omega, \mathscr{I}}(a, b)=\psi^{\mathscr{I}}(a, b)=\psi^{\mathscr{I}}(\alpha, \beta ; a, b)
$$

exists $\omega$-a.s. and is nonrandom.

$\mathrm{Pr}$ o o f. Since the polymer starts and ends at the interface, the proof can be done via a standard subadditivity argument in which two pieces of the polymer are concatenated (see, e.g., [4] or [27]). Indeed, fix $a$ and $b$. Then, for any $L_{1}$ and $L_{2}$,

$$
Z_{a\left(L_{1}+L_{2}\right), b\left(L_{1}+L_{2}\right)}^{\omega, \mathscr{\mathscr { I }}} \geqslant Z_{a L_{1}, b L_{1}}^{\omega, \mathscr{I}} Z_{a L_{2}, b L_{2}}^{\sigma^{a L_{1} \omega, \mathscr{I}}}
$$

where $\sigma$ is the left-shift acting on $\omega$. Define

$$
\Psi_{K}^{\omega, \mathscr{I}}(a, b)=\ln Z_{K,(b / a) K}^{\omega, \mathscr{I}} .
$$

Then, for any $K_{1}\left(=a L_{1}\right)$ and $K_{2}\left(=a L_{2}\right)$, we have

$$
\Psi_{K_{1}+K_{2}}^{\omega, \mathscr{I}}(a, b) \geqslant \Psi_{K_{1}}^{\omega, \mathscr{I}}(a, b)+\Psi_{K_{2}}^{\sigma^{K_{1} \omega, \mathscr{I}}}(a, b) .
$$


We can now apply Kingman's superadditive ergodic theorem, noting that $K^{-1} \Psi_{K}^{\omega, \mathscr{I}}(a, b)$ is bounded from above, to conclude that $\lim _{K \rightarrow \infty} K^{-1} \Psi_{K}^{\omega, \mathscr{J}}(a, b)=\psi^{\mathscr{J}}(a, b)$ exists $\omega$-a.s. and is nonrandom.

The relation linking $\psi_{A B}(a)$ to $\psi^{\mathscr{J}}(a, b)$ is the following.

Lemma 2.2.2. For all $(\alpha, \beta) \in \mathbf{R}^{2}$ and $a \geqslant 2$,

$$
\begin{aligned}
\psi_{A B}(a) & =\psi_{A B}(\alpha, \beta ; a) \\
& =\sup _{\substack{0 \leqslant b \leqslant 1, a_{1} \geqslant b, a_{2} \geqslant 2-b, a_{1}+a_{2}=a}} \frac{a_{1} \psi^{\mathscr{J}}\left(a_{1}, b\right)+a_{2}\left[\alpha / 2+\kappa\left(a_{2}, 1-b\right)\right]}{a_{1}+a_{2}} .
\end{aligned}
$$

$\mathrm{Pr}$ o of. The idea behind this relation is that the polymer follows the $A B$-interface over a distance $b L$ during $a_{1} L$ steps and then wanders away from the $A B$-interface to the diagonally opposite corner over a distance $(1-b) L$ during $a_{2} L$ steps. The optimal strategy is obtained by maximizing over $b, a_{1}$ and $a_{2}$ (recall Figure 4 ).

A formal proof goes as follows. Look at the last time $u$ and the last site $(v, 0)$ on the $A B$-interface before the polymer wanders off. This allows us to write the associated partition sum as

$$
Z_{A B}^{\omega}(a L, L)=\sum_{v=0}^{L} \sum_{u=v}^{a L-(2 L-v)} Z_{u, v}^{\omega, \mathscr{J}} Z_{a L-u, L-v}^{\sigma^{v} \omega},
$$

where $Z_{u, v}^{\omega, \mathscr{I}}$ is the partition sum for the single interface model to go in $u$ steps from $(0,0)$ to $(v, 0)$, and $Z_{a L-u, L-v}^{\sigma^{u} \omega}$ is the partition sum to go in $a L-u$ steps from $(v, 0)$ to $(L, L)$ without returning to the interface. Rewrite $(2.2 .7)$ as

$$
Z_{A B}^{\omega}(a L, L)=[1+o(1)] L^{2} \int_{0}^{1} d b \int_{b}^{a-(2-b)} d a_{1} Z_{a_{1} L, b L}^{\omega, \mathscr{I}} Z_{\left(a-a_{1}\right) L,(1-b) L}^{\sigma^{b L} \omega}
$$

From Lemma 2.1.1 and Lemma 2.2.1 we know that, as $L \rightarrow \infty$,

$$
\begin{aligned}
\frac{1}{L} \ln Z_{a_{1} L, b L}^{\omega, \mathscr{J}}= & {[1+o(1)] a_{1} \psi^{\mathscr{J}}\left(a_{1}, b\right) \quad \omega \text {-a.s., } } \\
\frac{1}{L} \ln Z_{\left(a-a_{1}\right) L,(1-b) L}^{\sigma^{b L} \omega}= & {[1+o(1)]\left(a-a_{1}\right) } \\
& \times\left(\frac{\alpha}{2}+\kappa\left(a-a_{1}, 1-b\right)\right) \quad \omega \text {-a.s. }
\end{aligned}
$$

For the latter, note that $\sigma^{b L} \omega$ changes with $L$. However, this causes no problem, because the distribution of $\omega$ is invariant under shifts and the shift length $b L$ is independent of $\omega$. Substitution of (2.2.9) into (2.2.8), and of the resulting expression into (1.3.1), yields the claim after we put $a_{2}=a-a_{1}$. Indeed, the right-hand sides of (2.2.9) are continuous in $b$ and $a_{1}$.

By obvious scaling, there exists a function $\phi^{\mathscr{G}}$ such that

$$
\psi^{\mathscr{J}}(a, b)=\phi^{\mathscr{F}}\left(\frac{a}{b}\right) \text {. }
$$

Therefore Lemma 2.2.2 yields the following. 
Proposition 2.2.2. For all $(\alpha, \beta) \in \mathbf{R}^{2}$,

$$
\begin{aligned}
S_{A B} & =\sup _{a \geqslant 2} \psi_{A B}(a) \\
& =\sup _{0 \leqslant b \leqslant 1, a_{1} \geqslant b, a_{2} \geqslant 2-b} \frac{a_{1} \phi^{\mathscr{J}}\left(a_{1} / b\right)+a_{2}\left[\alpha / 2+\kappa\left(a_{2}, 1-b\right)\right]}{a_{1}+a_{2}},
\end{aligned}
$$

P r o o f. Insert (2.2.10) into (2.2.6) and take the supremum over $a$.

This completes the identification of $S_{A B}$. The same formula applies for $S_{B A}$ but with $\alpha$ and $\beta$ interchanged, i.e.,

$$
S_{B A}(\alpha, \beta)=S_{A B}(\beta, \alpha) \text {. }
$$

Recall from the remark made below (1.3.2) that the first index labels the type of the block that is diagonally crossed, while the second index labels the type of the block that appears as its neighbor.

Note that $\phi^{\mathscr{J}}$ is symmetric in $\alpha$ and $\beta$. The asymmetry in (2.2.4), coming from the fact that the interface is labelled $B$ while the polymer starts at the interface, is not felt in the limit as $L \rightarrow \infty$. Further note that

$$
\begin{array}{rlrl}
\phi^{\mathscr{J}}(\alpha, \beta ; \mu) & \in\left[\frac{\alpha}{2}+\widehat{\kappa}(\mu), \alpha+\widehat{\kappa}(\mu)\right] \quad \forall \alpha \geqslant \beta \geqslant 0, \\
\phi^{\mathscr{J}}(\alpha, \beta ; \mu) & =\frac{\alpha}{2}+\widehat{\kappa}(\mu) & \forall \alpha \geqslant 0 \geqslant \beta .
\end{array}
$$

We close with the following facts.

Lemma 2.2.3. Let $k, l \in\{A, B\}$.

(i) For all $(\alpha, \beta) \in \mathbf{R}^{2}, a \mapsto a \psi_{k l}(\alpha, \beta ; a)$ is continuous and concave on $[2, \infty)$.

(ii) For all $a \in[2, \infty), \alpha \mapsto \psi_{k l}(\alpha, \beta ; a)$ and $\beta \mapsto \psi_{k l}(\alpha, \beta ; a)$ are continuous and nondecreasing on $\mathbf{R}$.

$\mathrm{P}$ r o of. (i) The claim is trivial for $k=l$, because of the simple form of $\psi_{A A}$ and $\psi_{B B}$ (recall (2.1.1) and (2.2.2)). The proof for $k \neq l$ runs as follows. Rewrite (2.2.6) as

$$
a \psi_{A B}(a)=\sup _{\substack{0 \leqslant b \leqslant 1, a_{1} \geqslant b, a_{2} \geqslant 2-b, a_{1}+a_{2}=a}}\left\{a_{1} \psi^{\mathscr{J}}\left(a_{1}, b\right)+a_{2}\left[\frac{1}{2} \alpha+\kappa\left(a_{2}, 1-b\right)\right]\right\} .
$$

From this it follows that

$$
\begin{aligned}
& \frac{1}{2} a^{1} \psi_{A B}\left(a^{1}\right)+\frac{1}{2} a^{2} \psi_{A B}\left(a^{2}\right) \\
& =\sup _{\substack{0 \leqslant b^{1} \leqslant 1, a_{1}^{1} \geqslant b^{1}, a_{2}^{1} \geqslant 2-b^{1}, a_{1}^{1}+a_{2}^{1}=a^{1}}} \sup _{\substack{0 \leqslant b^{2} \leqslant 1, a_{1}^{2} \geqslant b^{2}, a_{2}^{2} \geqslant 2-b^{2}, a_{1}^{2}+a_{2}^{2}=a^{2}}}\left\{\frac{1}{2} a_{1}^{1} \psi^{\mathscr{J}}\left(a_{1}^{1}, b^{1}\right)+\frac{1}{2} a_{1}^{2} \psi^{\mathscr{J}}\left(a_{1}^{2}, b^{2}\right)\right. \\
& \left.\quad+\frac{1}{2}\left(a_{2}^{1}+a_{2}^{2}\right) \frac{1}{2} \alpha+\frac{1}{2} a_{2}^{1} \kappa\left(a_{2}^{1}, 1-b^{1}\right)+\frac{1}{2} a_{2}^{2} \kappa\left(a_{2}^{2}, 1-b^{2}\right)\right\} .
\end{aligned}
$$


A standard concatenation argument gives

$$
\begin{aligned}
\frac{1}{2} a_{1}^{1} \psi^{\mathscr{I}}\left(a_{1}^{1}, b^{1}\right)+\frac{1}{2} a_{1}^{2} \psi^{\mathcal{I}}\left(a_{1}^{2}, b^{2}\right) & \leqslant \bar{a}_{1} \psi^{\mathscr{J}}\left(\bar{a}_{1}, \bar{b}\right), \\
\frac{1}{2} a_{2}^{1} \kappa\left(a_{2}^{1}, 1-b^{1}\right)+\frac{1}{2} a_{2}^{2} \kappa\left(a_{2}^{2}, 1-b^{2}\right) & \leqslant \bar{a}_{2} \kappa\left(\bar{a}_{2}, 1-\bar{b}\right),
\end{aligned}
$$

where we denote $\bar{a}_{1}=\left(a_{1}^{1}+a_{1}^{2}\right) / 2, \bar{a}_{2}=\left(a_{2}^{1}+a_{2}^{2}\right) / 2, \bar{b}=\left(b^{1}+b^{2}\right) / 2$. Since the double supremum in (2.2.15) is more restrictive than the single supremum over $0 \leqslant \bar{b} \leqslant 1, \bar{a}_{1} \geqslant \bar{b}, \bar{a}_{2} \geqslant 2-\bar{b}, \bar{a}_{1}+\bar{a}_{2}=\bar{a}$, with $\bar{a}=\left(a^{1}+a^{2}\right) / 2$, it follows from (2.2.6) and (2.2.15), (2.2.16) that $\frac{1}{2} a^{1} \psi_{A B}\left(a^{1}\right)+\frac{1}{2} a^{2} \psi_{A B}\left(a^{2}\right) \leqslant$ $\bar{a} \psi_{A B}(\bar{a})$. A similar argument applies to $\psi_{B A}$, after replacing $\frac{1}{2} \alpha$ by $\frac{1}{2} \beta$ and noting that $\psi^{\mathscr{I}}$ is symmetric in $\alpha$ and $\beta$.

(ii) The claim is again trivial for $k=l$. For $k \neq l$, note that $\psi^{\mathscr{J}}$ has the same property, as is obvious from (2.2.3)-(2.2.5). Hence the claim follows from Lemma 2.2.2.

2.3. Criterion for $S_{A B}>S_{A A}$. For all $(\alpha, \beta) \in \mathbf{R}^{2}$, we have $S_{A B} \geqslant S_{A A}$. The following gives us a criterion for when strict inequality occurs. In Section 4.1.1 this will be proved to be the criterion for localization when $p \geqslant p_{c}$.

Proposition 2.3.1. $S_{A B}>S_{A A}$ if and only if

$$
\sup _{\mu \geqslant 1} \mu\left[\phi^{\mathscr{I}}(\mu)-S_{A A}\right]>\frac{1}{2} \ln \frac{9}{5} \text {. }
$$

P r o of. From Propositions 2.2.1 and 2.2.2, together with the reparametrisation $\mu=a_{1} / b$ and $\nu=a_{2} / b$, it follows that

$$
S_{A B}-S_{A A}=\sup _{\mu \geqslant 1, \nu \geqslant 1} \frac{\mu\left[\phi^{\mathscr{I}}(\mu)-S_{A A}\right]-\nu\left[\frac{1}{2} \ln 5-f(\nu)\right]}{\mu+\nu}
$$

with

$$
f(\nu)=\sup _{2 /(\nu+1) \leqslant b \leqslant 1} \kappa(b \nu, 1-b), \quad \nu \geqslant 1 .
$$

Denote $g(\nu)=\nu\left[\frac{1}{2} \ln 5-f(\nu)\right]$. Below we will show that
(i) $g(\nu)>\frac{1}{2} \ln \frac{9}{5}$ for all $\nu \geqslant 1$
(ii) $\lim _{\nu \rightarrow \infty} g(\nu)=\frac{1}{2} \ln \frac{9}{5}$.

This will imply the claim as follows. If $\mu\left[\phi^{\mathscr{J}}(\mu)-S_{A A}\right] \leqslant \frac{1}{2} \ln \frac{9}{5}$ for all $\mu$, then by (i) the numerator in (2.3.1) is strictly negative for all $\mu$ and $\nu$, and so by (ii) the supremum is taken at $\nu=\infty$, resulting in $S_{A B}-S_{A A}=0$. On the other hand, if $\mu\left[\phi^{\mathscr{I}}(\mu)-S_{A A}\right]>\frac{1}{2} \ln \frac{9}{5}$ for some $\mu$, then, for that $\mu$, by (i) and (ii) the numerator is strictly positive for $\nu$ large enough, resulting in $S_{A B}-S_{A A}>0$. 
To prove (2.3.3), we will need the following inequality. Denote $\chi(a, b)=$ $a \kappa(a, b)$. Then by Lemma 2.1.1(ii) we have, for all $(s, t) \neq(u, v)$ in DOM,

$$
\begin{aligned}
\chi(s, t)-\chi(u, v) & =\int_{0}^{1} d w \frac{\partial}{\partial w} \chi(u+w(s-u), v+w(t-v)) \\
& >\left[\frac{\partial}{\partial w} \chi(u+w(s-u), v+w(t-v))\right]_{w=1} \\
& =(s-u)\left(\frac{\partial}{\partial a} \chi\right)(s, t)+(t-v)\left(\frac{\partial}{\partial b} \chi\right)(s, t) .
\end{aligned}
$$

To prove (2.3.3)(i), put $b=a / \nu$ in (2.3.2) and use Lemma 2.1.1(iv), (v) to rewrite the statement in $(2.3 .3)$ (i) as

$\kappa\left(a, 1-\frac{a}{\nu}\right)<\kappa\left(a^{*}, 1\right)-\frac{a^{*}}{\nu}\left(\frac{\partial}{\partial b} \kappa\right)\left(a^{*}, 1\right) \quad$ for all $\nu \geqslant 1$ and $\frac{2 \nu}{\nu+1} \leqslant a \leqslant \nu$.

But this inequality follows from (2.3.4) by choosing $s=a^{*}, t=1, u=a, v=$ $1-a / \nu$, cancelling a term $a^{*} \kappa\left(a^{*}, 1\right)$ on both sides, using that $\left(\frac{\partial}{\partial a} \kappa\right)\left(a^{*}, 1\right)=$ 0 , and afterwards cancelling a common factor $a$ on both sides.

To prove (2.3.3)(ii), we argue as follows. Choosing $b=a^{*} / \nu$ in (2.3.2), we get from Lemma 2.1.1(iv) that

$$
g(\nu) \leqslant \nu\left[\kappa\left(a^{*}, 1\right)-\kappa\left(a^{*}, 1-\frac{a^{*}}{\nu}\right)\right] .
$$

Letting $\nu \rightarrow \infty$, we get from Lemma 2.1.1(v) that

$$
\limsup _{\nu \rightarrow \infty} g(\nu) \leqslant a^{*}\left(\frac{\partial}{\partial b} \kappa\right)\left(a^{*}, 1\right)=\frac{1}{2} \ln \frac{9}{5} .
$$

Combine this with (2.3.3)(i) to get (2.3.3)(ii).

Proposition 2.3.1 says that the free energy per step for an $A B$-block exceeds that for an $A A$-block if and only the free energy per step for the single linear interface exceeds the free energy per step for an $A A$-block by a certain positive amount. This excess is needed to compensate for the loss of entropy that occurs when the path runs along the interface for awhile before moving upwards from the interface to end at the diagonally opposite corner (recall Figure 4). The constant $\frac{1}{2} \ln \frac{9}{5}$ is special to our model.

2.4. Criterion for $\psi_{A B}(\bar{x})>\psi_{A A}(\bar{x})$ and $\psi_{B A}(\bar{y})>\psi_{B B}(\bar{y})$. For all $(\alpha, \beta) \in \mathbf{R}^{2}$ and $a \geqslant 2$, we have

$$
\psi_{A B}(a) \geqslant \psi_{A A}(a), \quad \psi_{B A}(a) \geqslant \psi_{B B}(a) .
$$

The following gives a criterion for when strict inequality occurs and is the analogue of Proposition 2.3.1.

Proposition 2.4.1. For all $a \geqslant 2, \psi_{A B}(a)>\psi_{A A}(a)$ if and only if

$$
\sup _{\mu \geqslant 1} \mu\left[\phi^{\mathscr{J}}(\mu)-\frac{1}{2} \alpha-\frac{1}{2} \ln \left(\frac{a}{a-2}\right)\right]>\frac{1}{2} \ln \left[\frac{4(a-2)(a-1)^{2}}{a}\right] \text {. }
$$


P r o of. Return to Lemma 2.2.2. Fix $a \geqslant 2$. By (2.2.2), (2.2.6), and (2.2.10), we have

$$
\begin{aligned}
& \psi_{A B}(a)-\psi_{A A}(a) \\
& =\sup _{\substack{0 \leqslant b \leqslant 1, a_{1} \geqslant b, a_{2} \geqslant 2-b, a_{1}+a_{2}=a}}\left\{a_{1} \phi^{\mathscr{J}}\left(\frac{a_{1}}{b}\right)+a_{2}\left[\frac{\alpha}{2}+\kappa\left(a_{2}, 1-b\right)\right]-\left(a_{1}+a_{2}\right) \frac{\alpha}{2}\right. \\
& \left.-\left(a_{1}+a_{2}\right) \kappa\left(a_{1}+a_{2}, 1\right)\right\} \frac{1}{a_{1}+a_{2}} .
\end{aligned}
$$

The denominator is fixed. Put $\mu=a_{1} / b$ and rewrite the numerator as

$$
\begin{array}{r}
\mu b \phi^{\mathscr{I}}(\mu)+(a-\mu b)\left[\frac{\alpha}{2}+\kappa(a-\mu b, 1-b)\right]-a\left[\frac{\alpha}{2}+\kappa(a, 1)\right] \\
=\mu b \phi^{\mathscr{I}}(\mu)-\mu b \frac{\alpha}{2}-[a \kappa(a, 1)-(a-\mu b) \kappa(a-\mu b, 1-b)] .
\end{array}
$$

By choosing $s=a, t=1, u=a-\mu b, v=1-b$ in (2.3.4), we obtain that for all $\mu \geqslant 1$ and $0<b \leqslant 1$ with $(\mu-1) b \leqslant a-2$,

$$
\begin{aligned}
& a \kappa(a, 1)-(a-\mu b) \kappa(a-\mu b, 1-b) \\
& \quad>\mu b\left(\frac{\partial}{\partial a}(a \kappa)\right)(a, 1)+b\left(\frac{\partial}{\partial b}(a \kappa)\right)(a, 1) .
\end{aligned}
$$

Since the right-hand side of (2.4.5) is $b$ times the derivative at $b=0$ of the left-hand side, it follows that the difference in (2.4.4) is $\leqslant 0$ for all $0 \leqslant b \leqslant 1$ if and only if its derivative at $b=0$ is $\leqslant 0$. This derivative equals

$$
\mu \phi^{\mathscr{I}}(\mu)-\mu \frac{\alpha}{2}-\mu\left(\frac{\partial}{\partial a}(a \kappa)\right)(a, 1)-\left(\frac{\partial}{\partial b}(a \kappa)\right)(a, 1) .
$$

After substituting the expressions for $\kappa(a, 1),\left(\frac{\partial}{\partial a} \kappa\right)(a, 1)$ and $\left(\frac{\partial}{\partial b} \kappa\right)(a, 1)$ that we computed in Section 2.1 .1 (recall (2.1.1), (2.1.5), (2.1.6), (2.1.8), (2.1.9)), we find that (2.4.6) equals

$$
\mu\left[\phi^{\mathscr{J}}(\mu)-\frac{\alpha}{2}-\frac{1}{2} \ln \left(\frac{a}{a-2}\right)\right]-\frac{1}{2} \ln \left[\frac{4(a-2)(a-1)^{2}}{a}\right] .
$$

Hence we get the claim.

For $\psi_{B A}(a)>\psi_{B B}(a)$ the same criterion applies as in (2.4.2) with $\frac{1}{2} \alpha$ replaced by $\frac{1}{2} \beta$. (Recall that $\phi^{\mathscr{I}}$ is symmetric in $\alpha$ and $\beta$ by the remark made below (2.2.12).)

2.5. Analysis of $F(\rho)$. In this section we analyse the variational problem in (1.5.3).

Proposition 2.5.1. Let $(\alpha, \beta) \in \mathrm{CONE}$ and $\rho \in(0,1)$. Denote $C=$ $\alpha-\beta \geqslant 0$. The variational formula in (1.5.3) has unique maximizers $\bar{x}=$ $\bar{x}(C, \rho)$ and $\bar{y}=\bar{y}(C, \rho)$ satisfying:

(i) $2<\bar{y}<a^{*}<\bar{x}<\infty$ when $C>0$ and $\bar{x}=\bar{y}=a^{*}$ when $C=0$.

(ii) $u(\bar{x})>v(\bar{y})$ when $C>0$ and $u(\bar{x})=v(\bar{y})$ when $C=0$. 
(iii) $\rho \mapsto \bar{x}(C, \rho)$ and $\rho \mapsto \bar{y}(C, \rho)$ are analytic and strictly decreasing on $(0,1)$ for all $C>0$.

(iv) $C \mapsto \bar{x}(C, \rho)$ and $C \mapsto \bar{y}(C, \rho)$ are analytic and strictly increasing, respectively, strictly decreasing on $(0, \infty)$ for all $\rho \in(0,1)$.

(v) $A s \rho \uparrow 1, \bar{x}(C, \rho) \downarrow a^{*}$ and $\bar{y}(C, \rho) \downarrow 10 /\left(5-e^{-C}\right)$ for all $C \geqslant 0$.

(vi) $A s \rho \downarrow 0, \bar{x}(C, \rho) \uparrow 10 e^{-C} /\left(5 e^{-C}-1\right)$ and $\bar{y}(C, \rho) \uparrow a^{*}$ when $0 \leqslant C<$ $\ln 5$, while $\bar{x}(C, \rho) \uparrow \infty$ and $\bar{y}(C, \rho) \uparrow 2 /\left(1-e^{-C}\right)$ when $C \geqslant \ln 5$.

(vii) $A s C \uparrow \infty, \bar{x}(C, \rho) \uparrow \infty$ and $\bar{y}(C, \rho) \downarrow 2$ for all $\rho \in(0,1)$.

P r o o f. Fix $(\alpha, \beta) \in \mathrm{CONE}$ and $\rho \in(0,1)$. The supremum in (1.5.3) is attained at those $x, y$ that solve the equations

$$
\begin{aligned}
0= & -\ln 2+\frac{(1-\rho) y}{2}(\alpha-\beta)+\frac{(1-\rho) y}{2} \ln \left(\frac{x(y-2)}{y(x-2)}\right) \\
& -\rho \ln (x-2)-(1-\rho) \ln (y-2), \\
0= & -\ln 2+\frac{\rho x}{2}(\beta-\alpha)+\frac{\rho x}{2} \ln \left(\frac{y(x-2)}{x(y-2)}\right) \\
& -\rho \ln (x-2)-(1-\rho) \ln (y-2) .
\end{aligned}
$$

Multiplying the first relation by $\rho x$, the second relation by $(1-\rho) y$, and adding them up, we get

$$
0=-[\rho x+(1-\rho) y]\{\ln 2+\rho \ln (x-2)+(1-\rho) \ln (y-2)\} .
$$

Alternatively, subtracting the second relation from the first, we get

$$
0=\frac{1}{2}[\rho x+(1-\rho) y]\left\{(\alpha-\beta)+\ln \left(\frac{x(y-2)}{y(x-2)}\right)\right\} .
$$

Hence, $x, y$ solve the equations

$$
\begin{aligned}
& 0=\ln 2+\rho \ln (x-2)+(1-\rho) \ln (y-2), \\
& 0=(\alpha-\beta)+\ln \left(\frac{x(y-2)}{y(x-2)}\right) .
\end{aligned}
$$

These are two coupled equations depending on $\rho$, respectively, $C=\alpha-\beta$. Since the equations are linearly independent, their solution is unique.

(i) Let $\bar{x}$ and $\bar{y}$ denote a unique solution of (2.5.1). Clearly, $\bar{x}=\bar{y}=$ $a^{*}=\frac{5}{2}$ when $C=0$. Suppose that $C>0$. Then it follows from the second line of $(2.5 .1)$ that $\bar{x} /(\bar{x}-2)<\bar{y} /(\bar{y}-2)$, or $\bar{x}>\bar{y}$. Moreover, it follows from the first line of (2.5.1) that it is not possible to have $\bar{x}>\bar{y} \geqslant a^{*}$ or $\bar{y}<\bar{x} \leqslant a^{*}$. Consequently, $\bar{y}<a^{*}<\bar{x}$. The fact that $(\bar{x}, \bar{y}) \neq(\infty, 2)$ follows from (2.5.1) as well.

(ii) By (1.5.2),

$$
\begin{aligned}
u(\bar{x})-v(\bar{y})= & \frac{\alpha-\beta}{2}+\left(\frac{1}{\bar{x}}-\frac{1}{\bar{y}}\right) \ln 2+\frac{1}{2} \ln \left(\frac{\bar{x}}{\bar{y}}\right) \\
& -\frac{\bar{x}-2}{2 \bar{x}} \ln (\bar{x}-2)+\frac{\bar{y}-2}{2 \bar{y}} \ln (\bar{y}-2) .
\end{aligned}
$$


Using (2.5.1), we may rewrite $\alpha-\beta$ and $\ln 2$ in terms of $\bar{x}, \bar{y}, \rho$. This gives, after some cancellations,

$$
u(\bar{x})-v(\bar{y})=\left(\frac{\rho}{\bar{x}}+\frac{1-\rho}{\bar{y}}\right) \ln \left(\frac{\bar{x}-2}{\bar{y}-2}\right) .
$$

This is $>0$ when $C>0$, because then $\bar{x}>\bar{y}$, and is $=0$ when $C=0$, because then $\bar{x}=\bar{y}$.

(iii) The analyticity follows from the uniqueness of the solution of (2.5.1) and the implicit function theorem. From the second line of (2.5.1) it follows that $\rho \mapsto \bar{x}(C, \rho)$ and $\rho \mapsto \bar{y}(C, \rho)$ are either both nonincreasing or both nondecreasing. Differentiating the first line of (2.5.1) with respect to $\rho$, we get

$$
0=\ln \left(\frac{\bar{x}-2}{\bar{y}-2}\right)+\frac{\rho}{\bar{x}-2} \frac{\partial}{\partial \rho} \bar{x}+\frac{1-\rho}{\bar{y}-2} \frac{\partial}{\partial \rho} \bar{y} .
$$

Since $\bar{x}>\bar{y}$ when $C>0$, the sum of the last two terms is $<0$. Therefore it is not possible that $\frac{\partial}{\partial \rho} \bar{x}, \frac{\partial}{\partial \rho} \bar{y} \geqslant 0$. Hence $\frac{\partial}{\partial \rho} \bar{x}, \frac{\partial}{\partial \rho} \bar{y}<0$.

(iv) The analyticity again follows from the uniqueness of the solution of (2.5.1) and the implicit function theorem. From the first line of (2.5.1) it follows that $C \mapsto \bar{x}(C, \rho)$ and $C \mapsto \bar{y}(C, \rho)$ are either nondecreasing, respectively, nonincreasing or vice versa. Differentiating the second line of (2.5.1) with respect to $C=\alpha-\beta$, we get

$$
0=1-\frac{2}{\bar{x}(\bar{x}-2)} \frac{\partial}{\partial C} \bar{x}+\frac{2}{\bar{y}(\bar{y}-2)} \frac{\partial}{\partial C} \bar{y} .
$$

Since $\bar{x}, \bar{y}>2$, it is not possible that $\frac{\partial}{\partial C} \bar{x} \leqslant 0 \leqslant \frac{\partial}{\partial C} \bar{y}$. Hence $\frac{\partial}{\partial C} \bar{y}<0<$ $\frac{\partial}{\partial C} \bar{x}$.

(v) Denote $\Delta=e^{-C} \in(0,1]$. Since $\bar{y} \leqslant a^{*}$, it follows from the first line of (2.5.1) that $\bar{x} \downarrow a^{*}$ as $\rho \uparrow 1$. The second line of (2.5.1) therefore gives $\bar{y} /(\bar{y}-2) \uparrow 5 \Delta^{-1}$, i.e., $\bar{y} \downarrow 10 /(5-\Delta)$.

(vi) It follows from (2.5.1) that, as $\rho \downarrow 0$, either $\bar{x} \uparrow A \in\left(a^{*}, \infty\right), \bar{y} \uparrow a^{*}$ or $\bar{x} \uparrow \infty, \bar{y} \uparrow 2 /(1-\Delta)$. Since $\bar{y} \leqslant a^{*}=\frac{5}{2}$, the latter is possible only when $\Delta \leqslant \frac{1}{5}$. The former applies when $\Delta>\frac{1}{5}$, in which case $A=10 /\left(5-\Delta^{-1}\right)=$ $10 \Delta /(5 \Delta-1)$.

(vii) This is immediate from (2.5.1).

Lemma 2.5.1. $(\alpha, \beta) \mapsto F(\alpha, \beta ; \rho)$ is analytic on $\mathbf{R}^{2}$ for all $\rho \in(0,1)$.

$\mathrm{P}$ r o of. This is immediate from (1.5.2), (1.5.3), and Proposition 2.5.1(iv).

\section{Free energy of the polymer}

In Section 3.1 we prove Theorem 1.3.1. In Section 3.2 we analyse the set $\mathscr{R}(p)$ in (1.3.6) and the supremum $\rho^{*}(p)$ in (1.5.1). 
3.1. Proof of Theorem 1.3.1. (i) Write out the partition sum in (1.2.2) in terms of partition sums in successive blocks:

$$
\begin{aligned}
Z_{n, L_{n}}^{\omega, \Omega}=\sum_{N=1}^{n / 2 L_{n}} \sum_{\left(\Pi_{i}\right)_{i=1}^{N}} \sum_{u_{1}=2 L_{n}}^{\infty} \cdots \sum_{u_{N}=2 L_{n}}^{\infty}\left[\prod_{i=1}^{N-1} Z_{u_{i}}^{\sigma^{u_{1}+\cdots+u_{i-1}} \omega}\left(t^{\Omega}\left(\Pi_{i}\right)\right)\right] \\
\quad \times Z_{n-\left(u_{1}+\cdots+u_{N-1}\right)}^{\sigma_{1}+\cdots+u_{N-1} \omega}\left(\left(t^{\Omega}\left(\Pi_{i}\right)\right)\right. \\
\quad \times 1\left\{u_{1}+\cdots+u_{N-1} \leqslant n<u_{1}+\cdots+u_{N-1}+u_{N}\right\} .
\end{aligned}
$$

Here, $N$ counts the number of blocks traversed, $\sigma$ is the left-shift acting on $\omega, \Pi_{i}$ is the $i$-th step of the coarse-grained path, $u_{i}$ counts the number of steps spent in the $i$-th block diagonally traversed by $\Pi_{i}, t^{\Omega}\left(\Pi_{i}\right)$ labels the type of the $i$-th block in $\Omega$, and $Z_{u}^{\omega}(t)$ is the partition sum for spending $u$ steps in a block of type $t$. We want to derive the asymptotics of this expression as $n \rightarrow \infty$. For reasons of space the argument below is somewhat sketchy, but the technical details are easy to fill in.

First, for the computation we pretend that after $n$ steps the path has just completed traversing a block, i.e., we replace the indicator in (3.1.1) by

$$
1\left\{u_{1}+\cdots+u_{N}=n\right\} \text {. }
$$

The error made in doing so is at most a factor $O(n)$. Next, in (3.1.1) we insert a weight $2^{-N}$ under the sum over $\left(\Pi_{i}\right)_{i=1}^{N}$, scale $u_{i}$ by putting $v_{i}=u_{i} / L_{n}$, change the sum over $u_{i}$ to an integral over $v_{i}$, and insert a weight $e^{-\left(v_{i}-2\right)}$ under the integral. This gives

$$
\begin{aligned}
Z_{n, L_{n}}^{\omega, \Omega}= & O(n) \sum_{N=1}^{n / 2 L_{n}}\left(2^{N} e^{\left(n / L_{n}\right)-2 N} L_{n}^{N}\right) \\
& \times\left[\sum_{\left(\Pi_{i}\right)_{i=1}^{N}} 2^{-N} \int_{2}^{\infty} d v_{1} e^{-\left(v_{1}-2\right)} \cdots \int_{2}^{\infty} d v_{N} e^{-\left(v_{N}-2\right)}\right. \\
& \left.\times \prod_{i=1}^{N} Z_{v_{i} L_{n}}^{\sigma^{\left(v_{1}+\cdots v_{i-1}\right) L_{n}} \omega}\left(t^{\Omega}\left(\Pi_{i}\right)\right) 1\left\{v_{1}+\cdots+v_{N}=\frac{n}{L_{n}}\right\}\right],
\end{aligned}
$$

where the term between round brackets compensates for the insertion of the weights (and can be computed because of (3.1.2)), while roundoff errors (coming from turning sums into integrals) disappear into the error term. The factor between round brackets is $e^{o(n)}$ and therefore is negligible. The point of the rewrite in (3.1.3) is that the sum over $\left(\Pi_{i}\right)_{i=1}^{N}$ and the integrals over $v_{i}$ are normalized. Therefore we can now introduce two independent sequences of random variables, $\widehat{\Pi}=\left\{\widehat{\Pi}_{i}: i \in \mathbf{N}\right\}, \widehat{v}=\left\{\widehat{v}_{i}: i \in \mathbf{N}\right\}$, which describe a random uniform coarse-grained path, respectively, a random sequence of scaled times that are i.i.d. and $\operatorname{Exp}(1)$ distributed on $[2, \infty)$. In terms of 
these random variables we can rewrite (3.1.3) as

$$
\begin{aligned}
Z_{n, L_{n}}^{\omega, \Omega}=e^{o(n)} \sum_{N=1}^{n / 2 L_{n}}\langle & \prod_{i=1}^{N} Z_{\widehat{v}_{i} L_{n}}^{\sigma^{\left(\hat{v}_{1}+\cdots \widehat{v}_{i-1}\right) L_{n}} \omega}\left(t^{\Omega}\left(\widehat{\Pi}_{i}\right)\right) \\
& \left.\times 1\left\{\widehat{v}_{1}+\cdots+\widehat{v}_{N}=\frac{n}{L_{n}}\right\}\right\rangle,
\end{aligned}
$$

where $\langle\cdot\rangle$ denotes expectation with respect to $(\widehat{\Pi}, \widehat{v})$. As $L_{n} \rightarrow \infty$ we have, for every fixed realization of $(\widehat{\Pi}, \widehat{v})$,

$$
Z_{\widehat{v}_{i} L_{n}}^{\sigma^{\left(\hat{v}_{1}+\cdots+\widehat{v}_{i-1}\right) L_{n}} \omega}\left(t^{\Omega}\left(\widehat{\Pi}_{i}\right)\right)=\exp \left\{L_{n}[1+o(1)] \widehat{v}_{i} \psi_{t^{\Omega}\left(\widehat{\Pi}_{i}\right)}\left(\widehat{v}_{i}\right)\right\} \quad \omega \text {-a.s. }
$$

with $\psi_{k l}\left(\widehat{v}_{i}\right)$ the free energy per step in a $k l$-block, where the path spends $\widehat{v}_{i} L_{n}$ steps, defined in (1.3.2). Here we use that the distribution of $\omega$ is invariant under shifts and that $\left(\widehat{v}_{1}+\cdots+\widehat{v}_{i-1}\right) L_{n}$ is independent of $\omega$.

Because of (3.1.4) and (3.1.5), we are in a position to use large deviation theory (for background see, e.g., [16, Chapters I and II]). To that end, we introduce the empirical distribution $\mathscr{E}_{N}^{\Omega}=\mathscr{E}_{N}^{\Omega}(\widehat{\Pi}, \widehat{v})=N^{-1} \sum_{i=1}^{N} \delta_{\left(t^{\Omega}\left(\widehat{\Pi}_{i}\right), \widehat{v}_{i}\right)}$, where $\delta_{(t, v)}$ is the unit measure at $(t, v)$. This $\mathscr{E}_{N}^{\Omega}$ counts the frequency at which the $\left(t^{\Omega}\left(\widehat{\Pi}_{i}\right), \widehat{v}_{i}\right)$ assume values in the space $\Theta=\{A A, A B, B A, B B\} \times$ $[2, \infty)$ and is an element of $\mathscr{P}(\Theta)$, the set of probability distributions on $\Theta$. With the help of (3.1.5), we may rewrite (3.1.4) as

$$
\begin{aligned}
Z_{n, L_{n}}^{\omega, \Omega}=e^{o(n)} \sum_{N=1}^{n / 2 L_{n}}\langle & \exp \left\{N L_{n}[1+o(1)]\left(\mathscr{E}_{N}^{\Omega}, h_{1}\right)_{*}\right\} \\
& \left.\times 1\left\{\left(\mathscr{E}_{N}, h_{2}\right)_{*}=\frac{n}{N L_{n}}\right\}\right\rangle,
\end{aligned}
$$

where we introduce two functions on $\Theta, h_{1}(t, v)=v \psi_{t}(v)$ and $h_{2}(t, v)=v$ for $(t, v) \in \Theta$, and put $(\mu, h)_{*}=\int_{\Theta} h d \mu$ for $\mu \in \mathscr{P}(\Theta)$. At this point, our partition sum has been rewritten in terms of an expectation with respect to the empirical distribution $\mathscr{E}_{N}$ (which depends on $(\widehat{\Pi}, \widehat{v})$ ).

Our next step is to scale $N$ by putting $M=N L_{n} / n$ and to rewrite (3.1.6) as

$$
Z_{n, L_{n}}^{\omega, \Omega}=e^{o(n)} \int_{0}^{1 / 2} d M\left\langle\exp \left[n \frac{\left(\mathscr{E}_{N}^{\Omega}, h_{1}\right)_{*}}{\left(\mathscr{E}_{N}^{\Omega}, h_{2}\right)_{*}}\right] 1\left\{\left(\mathscr{E}_{N}^{\Omega}, h_{2}\right)_{*}=\frac{1}{M}\right\}\right\rangle,
$$

where again roundoff errors disappear into the error term. The idea that we now use is that $\left(\mathscr{E}_{N} \Omega\right)_{N \in \mathbf{N}}$ satisfies the large deviation principle on $\mathscr{P}(\Theta)$ with rate $N$ and with some rate function $\mu \mapsto I(\mu)$ that has compact level sets. This rate function will be $\Omega$-a.s. constant. For techniques on how to prove this, we refer to [8], [14], and [28]. Thus, the probability of $\mathscr{E}_{N}^{\Omega}$ being close to some $\mu \in \mathscr{P}(\Theta)$ is $\exp \{-N[1+o(1)] I(\mu)\}$. Since $N=M n / L_{n}=o(n)$, 
- we conclude that these large deviations have a negligible cost. Using (3.1.7), together with the relation

$$
\int_{0}^{1 / 2} d M 1\left\{\left(\mathscr{E}_{N}^{\Omega}, h_{2}\right)_{*}=\frac{1}{M}\right\}=\frac{1}{O(n)}
$$

(which follows from reversing the calculations above), we arrive at

$$
f=\lim _{n \rightarrow \infty} \frac{1}{n} \ln Z_{n, L_{n}}^{\omega, \Omega}=\sup _{\mu \in \mathscr{P}(\Theta)} \frac{\left(\mu, h_{1}\right)_{*}}{\left(\mu, h_{2}\right)_{*}} \quad \omega \text {-a.s. }
$$

But the supremum in the right-hand side is precisely the formula for $f$ stated in Theorem 1.3.1(i). The free energy is trivially finite (recall (1.2.1), (1.2.2) and (1.3.7)).

$\mathrm{R} \mathrm{e} \mathrm{m}$ a $\mathrm{r} \mathrm{k}$. In the above, somewhat sketchy, computation the introduction of the rate function can be avoided by appealing to concentration of measure estimates, which are a crude yet flexible form of large deviations (see [20] for an application of this technique in the context of self-avoiding random copolymers near a single linear interface).

(ii) The proof is elementary. Fix $\omega$ and $\Omega$, and rewrite the partition sum in (1.2.2) as

$$
Z_{n, L_{n}}^{\omega, \Omega}=\sum_{v_{A}, v_{B}} c_{n, L_{n}}^{\omega, \Omega}\left(v_{A}, v_{B}\right) e^{\alpha v_{A}+\beta v_{B}}
$$

with

$$
\begin{array}{r}
c_{n, L_{n}}^{\omega, \Omega}\left(v_{A}, v_{B}\right)=\mid\left\{\pi \in \mathscr{W}_{n, L_{n}}: \sum_{i=1}^{n} 1\left\{\omega_{i}=\Omega_{\pi_{i}}=A\right\}=v_{A},\right. \\
\left.\sum_{i=1}^{n} 1\left\{\omega_{i}=\Omega_{\pi_{i}}=B\right\}=v_{B}\right\} \mid .
\end{array}
$$

Choose any $\alpha_{1}, \alpha_{2}, \beta_{1}, \beta_{2} \in \mathbf{R}$. Then, by the Cauchy-Schwarz inequality applied to (3.1.7),

$$
Z_{n, L_{n}}^{\omega, \Omega}\left(\frac{\alpha_{1}+\alpha_{2}}{2}, \frac{\beta_{1}+\beta_{2}}{2}\right) \leqslant Z_{n, L_{n}}^{\omega, \Omega}\left(\alpha_{1}, \beta_{1}\right) Z_{n, L_{n}}^{\omega, \Omega}\left(\alpha_{2}, \beta_{2}\right)
$$

from which the claim follows (recall (1.2.2) and (1.3.7)).

(iii) According to Proposition 3.2.1(i) below, $p \mapsto \mathscr{R}(p)$ is continuous in the Hausdorff metric. It is therefore immediate from the variational representation of $f$ in (1.3.8), together with the continuity of $\psi_{k l}$ stated in Lemma 2.2.3(i), that $p \mapsto f(\alpha, \beta ; p)$ is continuous.

3.2. Analysis of $\mathscr{R}(p)$ and $\rho^{*}(p)$. The following proposition is crucial for the analysis of the phase transition curves. Recall (1.3.6), (1.5.1), and Figure 6. The elements of $\mathscr{R}(p)$ are matrices $\left(\begin{array}{cc}\rho_{A A} & \rho_{A B} \\ \rho_{B A} & \rho_{B B}\end{array}\right)$ whose entries are nonnegative and sum up to 1 . 
Proposition 3.2.1. (i) $p \mapsto \mathscr{R}(p)$ is continuous in the Hausdorff metric.

(ii) If $p \geqslant p_{c}$, then

$$
\left(\begin{array}{cc}
1-\gamma & \gamma \\
0 & 0
\end{array}\right) \in \mathscr{R}(p) \quad \text { for some } \quad \gamma \in(0,1) .
$$

(iii) If $p<p_{c}$, then

$$
\left(\begin{array}{cc}
1-\gamma & \gamma \\
0 & 0
\end{array}\right) \notin \mathscr{R}(p) \quad \text { for all } \quad \gamma \in[0,1] \text {. }
$$

P r o o f. (i) Return to (1.3.3)-(1.3.6). Choose $0<p<p^{\prime}<1$. Let $\Omega$ and $\Omega^{\prime}$ be two typical percolation configurations with parameter $p$ and $p^{\prime}$, respectively, coupled such that the set of $A$ 's in $\Omega^{\prime}$ contains the set of $A$ 's in $\Omega$. Then

$$
\begin{gathered}
\limsup _{n \rightarrow \infty}\left|\rho_{k l}^{\Omega}(\Pi, n)-\rho_{k l}^{\Omega}(\Pi, n)\right| \leqslant 2\left(p^{\prime}-p\right) \quad \Omega, \Omega^{\prime} \text {-a.s. } \\
\forall k, l \in\{A, B\}, \quad \Pi \in \mathscr{W} .
\end{gathered}
$$

Therefore, for all $k, l \in\{A, B\}$, we have $\left|\left(\mathscr{R}^{\Omega}-\mathscr{R}^{\Omega^{\prime}}\right)_{k l}\right| \leqslant 2\left(p^{\prime}-p\right) \Omega, \Omega^{\prime}$-a.s. and hence $\left|\left[\mathscr{R}(p)-\mathscr{R}\left(p^{\prime}\right)\right]_{k l}\right| \leqslant 2\left(p^{\prime}-p\right)$.

(ii) If $p>p_{c}$, then there is an infinite cluster of $A$-blocks with a strictly positive density. For the coarse-grained path $\Pi$ that moves to the infinite cluster and afterwards stays inside this cluster, we have $\rho_{A}=\rho_{A A}+\rho_{A B}=1$. For the coarse-grained path $\Pi$ that moves to the infinite cluster, afterwards stays inside this cluster, but follows its boundary as much as possible, we have $0<\rho_{A B}=1-\rho_{A A}<1$, which proves the claim. Since $p \mapsto \mathscr{R}(p)$ is continuous in the Hausdorff metric, and since lowering $p$ increases the density of the $B$ blocks, the claim trivially extends to $p=p_{c}$.

(iii) If $p<p_{c}$, then there is no infinite cluster of $A$-blocks. In fact, any coarse-grained path $\Pi$ visits $B$-blocks with a strictly positive density (as follows from a coupling argument similar as in (i)). Hence, $\rho_{A}=\rho_{A A}+\rho_{A B}<1$ for all $\Pi$, and in fact $\sup _{\Pi \in \mathscr{W}} \rho_{A}(\Pi)<1$. Since $\mathscr{R}(p)$ is a closed set, this proves the claim.

It follows from Proposition 3.2.1 and the fact that $\mathscr{R}(p)$ is closed that $\rho^{*}(p)$ defined in (1.5.1) has the qualitative properties indicated in Figure 6: $p \mapsto \rho^{*}(p)$ is continuous and nondecreasing on $(0,1), \rho^{*}(p)=1$ for $p \in\left[p_{c}, 1\right)$ and $\rho^{*}(p) \in(0,1)$ for $p \in\left(0, p_{c}\right)$.

\section{Analysis of the critical curve}

In Section 4.1 we prove Theorems 1.4.1, 1.4.2, and 1.4.3 for $p \geqslant p_{c}$. In Section 4.2 we prove Theorems 1.5.1, 1.5.2, and 1.5.3 for $p<p_{c}$. In Section 4.3 we make some observations about the separation of the localised phase into two subphases for $p<p_{c}$. 


\subsection{Supercritical case $p \geqslant p_{c}$.}

4.1.1. Proof of Theorems 1.4 .1 and 1.4.2. The following proposition proves Theorems 1.4.1 and 1.4.2. Recall (Section 2.3) that $S_{A B} \geqslant S_{A A}$.

Proposition 4.1.1. Fix $p \geqslant p_{c}$.

(i) If $S_{A B}=S_{A A}$, then $f=S_{A A}$.

(ii) If $S_{A B}>S_{A A}$, then $f>S_{A A}$.

P r o o f. The proof uses Theorem 1.3.1(i) and Proposition 3.2.1(ii), in combination with the inequalities

$$
S_{B B} \leqslant S_{A A}, \quad S_{B A} \leqslant S_{A B},
$$

which hold since $\beta \leqslant \alpha$ (recall Propositions 2.2.1 and 2.2.2).

(i) Suppose that $S_{A B}=S_{A A}$. Then, since $\psi_{k l}(a) \leqslant S_{k l}$ for all $a \geqslant 2$ and $k, l \in\{A, B\}$ by (1.4.4), Theorem 1.3.1(i) and (4.1.1) yield

$$
f \leqslant \sup _{\left(a_{k l}\right) \in \mathscr{A}} \sup _{\left(\rho_{k l}\right) \in \mathscr{R}(p)} \frac{\sum_{k, l} \rho_{k l} a_{k l} S_{k l}}{\sum_{k, l} \rho_{k l} a_{k l}} \leqslant \sup _{k, l} S_{k l}=S_{A B}=S_{A A} .
$$

On the other hand, Theorem 1.3.1(i) and Proposition 3.2.1(ii)yield

$$
f \geqslant \frac{(1-\gamma) \bar{a}_{A A} S_{A A}+\gamma \bar{a}_{A B} S_{A B}}{(1-\gamma) \bar{a}_{A A}+\gamma \bar{a}_{A B}}=S_{A A},
$$

where $\bar{a}_{A A}, \bar{a}_{A B}$ are the maximizers of $S_{A A}, S_{A B}$ (and the value of $\gamma$ is irrelevant). Combine (4.1.2) and (4.1.3) to get $f=S_{A A}$.

(ii) Suppose that $S_{A B}>S_{A A}$. Then Theorem 1.3.1(i) and Proposition 3.2.1(ii) with $0<\gamma<1$ yield

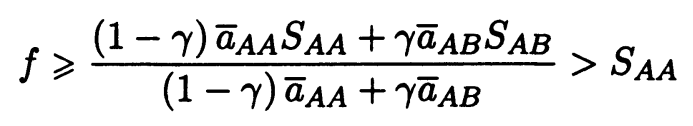

(here it is important that $\gamma>0$ ).

We see from Proposition 4.1.1 that $\mathscr{D}$ corresponds to the situation where the polymer is fully $A$-delocalized $\left(f=S_{A A}\right.$ ), while $\mathscr{L}$ corresponds to the situation where the polymer is partially $A B$-delocalized $\left(S_{A A}<f<S_{A B}\right)$.

4.1.2. Proof of Theorem 1.4.3. Since $S_{A A}(\alpha, \beta)$ does not depend on $\beta$, and $\beta \mapsto S_{A B}(\alpha, \beta)$ is continuous and nondecreasing on $\mathbf{R}$ for every $\alpha \in \mathbf{R}$ by Lemma 2.2.3(ii), the boundary between $\mathscr{D}$ and $\mathscr{L}$ is a continuous function in CONE. We denote this function by $\alpha \mapsto \beta_{c}(\alpha)$.

We first show that the curve is concave. To that end, choose any $\alpha_{1}<\alpha_{2}$, and consider the points $\left(\alpha_{1}, \mathcal{L}_{c}\left(\alpha_{1}\right)\right)$ and $\left(\alpha_{2}, \beta_{c}\left(\alpha_{2}\right)\right)$ on the curve. Let $\left(\alpha_{3}, \beta_{3}\right)$ be the midpoint of the line connecting the two. We want to show that $\beta_{3} \leqslant \beta_{c}\left(\alpha_{3}\right)$. By the convexity of $f$, stated in Theorem 1.3.1(ii), we have

$$
f\left(\alpha_{3}, \beta_{3}\right) \leqslant \frac{1}{2}\left[f\left(\alpha_{1}, \beta_{c}\left(\alpha_{1}\right)\right)+f\left(\alpha_{2}, \beta_{c}\left(\alpha_{2}\right)\right)\right]
$$


Since the curve itself is part of $\mathscr{D}$ (recall (1.4.3)), it follows from Propositions 2.2.1 and 4.1.1(i) that the right-hand side of (4.1.5) equals

$$
\frac{1}{2}\left[\left(\frac{1}{2} \alpha_{1}+\kappa\right)+\left(\frac{1}{2} \alpha_{2}+\kappa\right)\right]=\frac{1}{2}\left(\frac{\alpha_{1}+\alpha_{2}}{2}\right)+\kappa=\frac{1}{2} \alpha_{3}+\kappa .
$$

Thus, $f\left(\alpha_{3}, \beta_{3}\right) \leqslant \frac{1}{2} \alpha_{3}+\kappa$. But, by Proposition 4.1.1, the reverse inequality is true always, and so equality holds. Consequently, $\left(\alpha_{3}, \beta_{3}\right) \in \mathscr{D}$, which proves the claim that $\beta_{3} \leqslant \beta_{c}\left(\alpha_{3}\right)$.

The concavity in combination with the lower bound in part (i) of the following lemma show that the curve is nondecreasing.

The following lemma settles most of Theorem 1.4.3.

Lemma 4.1.1. Fix $p \geqslant p_{c}$.

(i) $\beta_{c}(\alpha) \geqslant \ln \left(2-e^{-\alpha}\right)$ for all $\alpha \geqslant 0$.

(ii) $\beta_{c}(\alpha)<8 \ln 3$ for all $\alpha \geqslant 0$.

(iii) $\beta_{c}(\alpha)=\alpha$ for all $0 \leqslant \alpha \leqslant \alpha_{0}$, where $\alpha_{0}$ is the number defined in the first line of (2.1.15).

P r o o f. (i) We have, recalling (2.2.3), (2.2.4),

$$
\phi^{\mathscr{I}}(\mu)=\lim _{L \rightarrow \infty} \frac{1}{L} \ln Z_{L}^{\omega, \mathscr{I}}(\mu) \quad \omega \text {-a.s. }
$$

with

$$
\begin{aligned}
& Z_{L}^{\omega, \mathscr{I}}(\mu)=\sum_{\pi \in \mathscr{W}_{\mu L, L}} \exp \left[-H_{\mu L}^{\omega, \mathscr{I}}(\pi)\right] \\
& H_{\mu L}^{\omega, \mathscr{I}}(\pi)=-\sum_{i=1}^{\mu L}\left(\alpha 1\left\{\omega_{i}=A, \pi_{i}>0\right\}+\beta 1\left\{\omega_{i}=B, \pi_{i} \leqslant 0\right\}\right) .
\end{aligned}
$$

We will derive an upper bound on $\phi^{\mathscr{J}}(\mu)$ by doing a so-called first-order annealed estimate (also referred to as a first-order Morita approximation; see [25]). This estimate consists in writing

$H_{\mu L}^{\omega, \mathscr{J}}(\pi)=-\sum_{i=1}^{\mu L} \alpha 1\left\{\omega_{i}=A\right\}-\sum_{i=1}^{\mu L} 1\left\{\pi_{i} \leqslant 0\right\}\left(-\alpha 1\left\{\omega_{i}=A\right\}+\beta 1\left\{\omega_{i}=B\right\}\right)$,

using that the first term is $-\mu L \frac{1}{2} \alpha[1+o(1)] \omega$-a.s. as $L \rightarrow \infty$ and is independent of $\pi$, substituting this into (4.1.6) and performing the expectation over $\omega$. This gives

$$
\begin{aligned}
\left\langle\ln Z_{L}^{\omega, \mathscr{I}}(\mu)\right\rangle \leqslant & \mu L \frac{1}{2} \alpha[1+o(1)] \\
& +\ln \sum_{\pi \in \mathscr{W}_{\mu L, L}} \prod_{i=1}^{\mu L} 1\left\{\pi_{i} \leqslant 0\right\}\left\langle e^{-\alpha 1\left\{\omega_{i}=A\right\}+\beta 1\left\{\omega_{i}=B\right\}}\right\rangle \\
\leqslant & \mu L \frac{1}{2} \alpha[1+o(1)]+\mu L[\widehat{\kappa}(\mu)+o(1)]+\mu L \ln \left(\frac{1}{2} e^{-\alpha}+\frac{1}{2} e^{\beta}\right),
\end{aligned}
$$


where $\langle\cdot\rangle$ denotes expectation over $\omega$, we use Jensen's inequality as well as the i.i.d. property of $\omega$ in the first line, and we use Lemma 2.1.2(i) in the second line. Consequently,

$$
\begin{aligned}
\phi^{\mathscr{I}}(\mu) & =\lim _{L \rightarrow \infty} \frac{1}{\mu L}\left\langle\ln Z_{L}^{\omega, \mathscr{I}}(\mu)\right\rangle \\
& \leqslant \frac{1}{2} \alpha+\widehat{\kappa}(\mu)+\ln \left(\frac{1}{2} e^{-\alpha}+\frac{1}{2} e^{\beta}\right) .
\end{aligned}
$$

Suppose that

$$
\ln \left(\frac{1}{2} e^{-\alpha}+\frac{1}{2} e^{\beta}\right) \leqslant 0 .
$$

Then substitution of (4.1.6) into (2.3.1) gives

$$
S_{A B}-S_{A A} \leqslant \sup _{\mu \geqslant 1, \nu \geqslant 1} \frac{\mu\left[\widehat{\kappa}(\mu)-\frac{1}{2} \ln 5\right]-\nu\left[\frac{1}{2} \ln 5-f(\nu)\right]}{\mu+\nu} .
$$

But the right-hand side is the same as $S_{A B}-S_{A A}$ when $\alpha=\beta=0$ (as can be seen from (2.3.1) because $\phi^{\mathscr{J}}(\mu)=\widehat{\kappa}(\mu)$ when $\alpha=\beta=0$ ), and therefore is equal to 0 . Hence, recalling (1.3.5), we find that (4.1.7) implies that $(\alpha, \beta) \in \mathscr{D}$. Consequently, $\ln \left(\frac{1}{2} e^{-\alpha}+\frac{1}{2} e^{\beta_{c}(\alpha)}\right) \geqslant 0$ for all $\alpha \geqslant 0$, which gives the lower bound that is claimed.

(ii) We will show that there exists a $\mu_{0}>1$ such that

$$
\mu_{0}\left[\phi^{\mathscr{J}}\left(\mu_{0}\right)-S_{A A}\right]>\frac{1}{2} \ln \frac{9}{5} \quad \text { for all } \alpha \geqslant 0 \text { when } \beta \geqslant 8 \ln 3 \text {. }
$$

This will prove the claim via Theorem 1.4.2 and Proposition 2.3.1.

Consider the polymer along the single infinite interface $\mathscr{I}$ introduced in Section 2.2.2. Fix $\omega$. In $\omega$, look for the strings of $B$ 's that are followed by a string of at least three $A$ 's. Call these $B$-strings «good», and call all other $B$-strings «bad». Let $\pi(\omega)$ be the path that starts at $(0,0)$, steps to $(0,1)$ and proceeds as follows. Each time a good $B$-string comes up, the path moves down from height 1 to height 0 during the step that carries the $A$ just preceding the good $B$-string, moves at height 0 during the steps that carry the $B$ 's inside the string, moves up from height 0 to height 1 during the step that carries the first $A$ after the string, and moves at height 1 during the step that carries the second $A$ after the string. The third $A$ can be used to either move from height 1 to height 0 in case the next good $B$-string comes up immediately, or to move at height 0 in case it is not. When a bad $B$-string comes up, the path stays at height 1.

Along $\pi(\omega)$, we have that all the $A$ 's lie in the upper halfplane, all the bad $B$-strings lie in the upper halfplane, while all the good $B$-strings lie in the interface. Asymptotically, the good $B$-strings contain $\frac{1}{4}$-th of the $B$ 's. Hence $\frac{1}{8}$-th of the steps carry a $B$ that is in a good $B$-string. Moreover, the number of steps between heights 0 and 1 is $\frac{1}{8}$ times the number of steps at 
heights 0 and 1 (the average length of a good $B$-string is 2), and so $\pi(\omega)$ travels a distance $L$ in time $\frac{9}{8} L$ for $L$ large, which corresponds to $\mu=\mu_{0}=\frac{9}{8}$. Thus, the contribution of $\pi(\omega)$ to the Hamiltonian in (2.2.4) equals

$$
H_{\mu_{0} L}^{\omega, \mathscr{J}}(\pi(\omega))=-\mu_{0} L\left(\frac{1}{2} \alpha+\frac{1}{8} \beta\right)[1+o(1)] \quad \omega \text {-a.s. as } L \rightarrow \infty \text {. }
$$

Therefore, recalling (2.2.5) and (2.2.10), we have $\phi^{\mathscr{J}}\left(\mu_{0}\right) \geqslant \frac{1}{2} \alpha+\frac{1}{8} \beta$. Via (2.2.1) this gives

$$
\mu_{0}\left[\phi^{\mathscr{J}}\left(\mu_{0}\right)-S_{A A}\right] \geqslant \mu_{0}\left(\frac{1}{8} \beta-\frac{1}{2} \ln 5\right) .
$$

Consequently, the inequality in (4.1.8) holds as soon as $\frac{1}{8} \beta>\frac{8}{9} \ln 3+\frac{1}{18} \ln 5$. Since the right-hand side is strictly smaller than $\ln 3$, this proves the claim.

(iii) Choose $\alpha=\beta$. Then, by (2.2.13), $\phi^{I}(\mu) \leqslant \alpha+\widehat{\kappa}(\mu)$. If $\alpha \in\left[0, \alpha_{0}\right]$, with $\alpha_{0}$ given by the first line of (2.1.15), then this bound in combination with Propositions 2.2.1 and 2.3.1 gives $S_{A B}=S_{A A}$. Thus,

$$
\left\{(\alpha, \alpha): \alpha \in\left[0, \alpha_{0}\right]\right\} \subset \mathscr{D} \text {. }
$$

Lemma 4.1.1, together with the concavity of $\alpha \mapsto \beta_{c}(\alpha)$ (shown prior to Lemma 4.1.1), proves Theorem 1.4.3, except for the slope discontinuity stated in (1.4.5). But the latter follows from the fact that if the piece of $\beta_{c}$ on $\left[\alpha^{*}, \infty\right)$ is analytically continued outside CONE, then it hits the vertical axis at a strictly positive value, namely, $\alpha_{0}$ defined in the first line of (2.1.15). Indeed, for $\alpha=0$ we have $\phi^{\mathscr{J}}(\mu)=\frac{1}{2} \beta+\widehat{\kappa}(\mu)$, because there is zero exponential cost for the path to stay in the lower halfplane (recall (2.2.3)-(2.2.5) and (2.2.10)). Consequently, the criterion for delocalization in Proposition 2.3.1, $S_{A B}=S_{A A}$, reduces to

$$
\sup _{\mu \geqslant 1} \mu\left[\widehat{\kappa}(\mu)+\frac{1}{2} \beta-\frac{1}{2} \ln 5\right] \leqslant \frac{1}{2} \ln \frac{9}{5} .
$$

This is true precisely when $\beta \leqslant \alpha_{0}$.

\subsection{Subcritical case $p<p_{c}$.}

\subsubsection{Proof of Theorems 1.5.1 and 1.5.2.}

Proposition 4.2.1. If $\psi_{A B} \equiv \psi_{A A}$ and $\psi_{B A} \equiv \psi_{B B}$, then $f=$ $F\left(\rho^{*}(p)\right)$.

P r o of. Suppose that $(\alpha, \beta)$ is such that $\psi_{A B}(a)=\psi_{A A}(a)$ and $\psi_{B A}(a)=\psi_{B B}(a)$ for all $a \geqslant 2$. Then the variational formula in (1.3.8) reduces to

$$
\begin{aligned}
f=\sup _{\left(a_{k l}\right) \in \mathscr{A}} \sup _{\left(\rho_{k l}\right) \in \mathscr{R}(p)}[ & {\left[\rho_{A A} a_{A A} \psi_{A A}\left(a_{A A}\right)+\rho_{A B} a_{A B} \psi_{A A}\left(a_{A B}\right)\right.} \\
& \left.+\rho_{B A} a_{B A} \psi_{B B}\left(a_{B A}\right)+\rho_{B B} a_{B B} \psi_{B B}\left(a_{B B}\right)\right] \\
& \times\left(\rho_{A A} a_{A A}+\rho_{A B} a_{A B}+\rho_{B A} a_{B A}+\rho_{B B} a_{B B}\right)^{-1}
\end{aligned}
$$


Define

$$
\tilde{a}_{A A}=\frac{\rho_{A A}}{\rho_{A}} a_{A A}+\frac{\rho_{A B}}{\rho_{A}} a_{A B}, \quad \tilde{a}_{B B}=\frac{\rho_{B A}}{\rho_{B}} a_{B A}+\frac{\rho_{B B}}{\rho_{B}} a_{B B}
$$

where $\rho_{A}=\rho_{A A}+\rho_{A B}$ and $\rho_{B}=\rho_{B A}+\rho_{B B}$. By Lemma 2.1.1(ii) and (2.2.2), $a \mapsto a \psi_{A A}(a)$ and $a \mapsto a \psi_{B B}(a)$ are concave on $[2, \infty)$. Hence, the numerator in (4.2.1) can be bounded above as

$$
\begin{aligned}
\rho_{A} & {\left[\frac{\rho_{A A}}{\rho_{A}} a_{A A} \psi_{A A}\left(a_{A A}\right)+\frac{\rho_{A B}}{\rho_{A}} a_{A B} \psi_{A A}\left(a_{A B}\right)\right] } \\
& +\rho_{B}\left[\frac{\rho_{B A}}{\rho_{B}} a_{B A} \psi_{B B}\left(a_{B A}\right)+\frac{\rho_{B B}}{\rho_{B}} a_{B B} \psi_{B B}\left(a_{B B}\right)\right] \\
\leqslant & \rho_{A} \tilde{a}_{A A} \psi_{A A}\left(\tilde{a}_{A A}\right)+\rho_{B} \tilde{a}_{B B} \psi_{B B}\left(\tilde{a}_{B B}\right),
\end{aligned}
$$

while the denominator in (4.2.1) equals $\rho_{A} \tilde{a}_{A A}+\rho_{B} \tilde{a}_{B B}$. For any choice of $\left(\rho_{k l}\right) \in \mathscr{R}(p)$, as $\left(a_{k l}\right)$ runs through $\mathscr{A}, \tilde{a}_{A A}$ and $\tilde{a}_{B B}$ run through all values $\geqslant 2$. Moreover, equality can be achieved in (4.2.3) by choosing $a_{A A}=a_{A B}$ and $a_{B A}=a_{B B}$. Hence (4.2.1) reduces to

$$
f=\sup _{\left(\rho_{k l}\right) \in \mathscr{R}(p)} F\left(\rho_{A}\right)
$$

with $F(\rho)$ given by (1.5.3). Thus, it remains to show that the supremum is taken at $\rho^{*}(p)=\sup _{\left(\rho_{k l}\right) \in \mathscr{R}(p)} \rho_{A}$.

For $\rho \in(0,1)$, let $\bar{x}=\bar{x}(\rho)$ and $\bar{y}=\bar{y}(\rho)$ denote the unique maximizers of (1.5.3). Then, for any $\rho_{1}, \rho_{2} \in(0,1)$,

$$
F\left(\rho_{1}\right)-F\left(\rho_{2}\right)=R\left(\rho_{1}, \bar{x}\left(\rho_{1}\right), \bar{y}\left(\rho_{1}\right)\right)-R\left(\rho_{2}, \bar{x}\left(\rho_{2}\right), \bar{y}\left(\rho_{2}\right)\right)
$$

with

$$
R(\rho, x, y)=\frac{\rho x u(x)-(1-\rho) y u(y)}{\rho x+(1-\rho) y} .
$$

Since $R\left(\rho_{1}, \bar{x}\left(\rho_{1}\right), \bar{y}\left(\rho_{1}\right)\right) \geqslant R\left(\rho_{1}, \bar{x}\left(\rho_{2}\right), \bar{y}\left(\rho_{2}\right)\right)$, we have

$$
\frac{\partial}{\partial \rho} F(\rho) \geqslant\left(\frac{\partial}{\partial \rho} R\right)(\rho, \bar{x}(\rho), \bar{y}(\rho))
$$

Now compute

$$
\left(\frac{\partial}{\partial \rho} R\right)(\rho, x, y)=\frac{x y[u(x)-v(y)]}{[\rho x+(1-\rho) y]^{2}}
$$

and use Proposition 2.5.1(ii), to conclude from (4.2.7) that $\frac{\partial}{\partial \rho} F(\rho)>0$. Hence (4.2.4) reduces to $F\left(\rho^{*}(p)\right)$.

The following proposition is the analogue of Proposition 4.1.1.

Proposition 4.2.2. Fix $p<p_{c}$.

(i) If $\psi_{A B}(\bar{x})=\psi_{A A}(\bar{x})$ and $\psi_{B A}(\bar{y})=\psi_{B B}(\bar{y})$, then $f=F\left(\rho^{*}(p)\right)$.

(ii) If $\psi_{A B}(\bar{x})>\psi_{A A}(\bar{x})$ or $\psi_{B A}(\bar{y})>\psi_{B B}(\bar{y})$, then $f . F\left(\rho^{*}(p)\right)$. 
$\mathrm{Pr}$ o o f. It follows from (1.3.8) and (2.4.1) that $f$ is bounded from below by the right-hand side of (4.2.1). The latter equals $F\left(\rho^{*}(p)\right)$, as shown in the proof of Proposition 4.2.1, and so

$$
f \geqslant F\left(\rho^{*}(p)\right) \text {. }
$$

(i) Denote $\theta_{k l}(a)=a \psi_{k l}(a)$. We know that $\theta_{A B} \geqslant \theta_{A A}$ and $\theta_{B A} \geqslant \theta_{B B}$ (by (2.4.1)), and that all four functions are concave (by Lemma 2.2.3(i)). Since $\theta_{A A}$ and $\theta_{B B}$ are both differentiable (by (2.1.1) and (2.2.2)), the assumption of equality at $\bar{x}$, respectively, $\bar{y}$ implies that $\theta_{A B}$ and $\theta_{B A}$ are differentiable at $\bar{x}$, respectively, $\bar{y}$ and that the equality carries over to the derivatives. Thus, we have

$$
\theta_{A B}^{\prime}(\bar{x})=\theta_{A A}^{\prime}(\bar{x}) \text { and } \theta_{B A}^{\prime}(\bar{y})=\theta_{B B}^{\prime}(\bar{y}) .
$$

Fix $\left(\rho_{k l}\right) \in \mathscr{A}$. Denote $\bar{N}=\rho_{A} \theta_{A A}(\bar{x})+\rho_{B} \theta_{B B}(\bar{y})$ and $\bar{D}=\rho_{A} \bar{x}+\rho_{B} \bar{y}$. The fact that $\bar{x}$ and $\bar{y}$ are the maximizers of

$$
\sup _{a_{A A}, a_{B B} \geqslant 2} \frac{\rho_{A} \theta_{A A}\left(a_{A A}\right)+\rho_{B} \theta_{B B}\left(a_{B B}\right)}{\rho_{A} a_{A A}+\rho_{B} a_{B B}}
$$

implies that $\theta_{A A}^{\prime}(\bar{x})=\theta_{B B}^{\prime}(\bar{y})=\bar{N} / \bar{D}$. Hence, all four derivatives in (4.2.10) are equal to $\bar{N} / \bar{D}$. Next, denote

$$
N=\sum_{k l} \rho_{k l} \theta_{k l}\left(a_{k l}\right) \quad \text { and } \quad D=\sum_{k l} \rho_{k l} a_{k l} .
$$

By the concavity of $a \mapsto \theta_{k l}(a)$ (recall Lemma 2.2.3(i)), we have that for all $\left(a_{k l}\right)$,

$$
\begin{aligned}
N \leqslant & \bar{N}+\rho_{A A}\left(a_{A A}-\bar{x}\right) \theta_{A A}^{\prime}(\bar{x})+\rho_{A B}\left(a_{A B}-\bar{x}\right) \theta_{A B}^{\prime}(\bar{x}) \\
& +\rho_{B A}\left(a_{B A}-\bar{y}\right) \theta_{B A}^{\prime}(\bar{y})+\rho_{B B}\left(a_{B B}-\bar{y}\right) \theta_{B B}^{\prime}(\bar{y})=\bar{N}+V \frac{\bar{N}}{\bar{D}}
\end{aligned}
$$

where $V=\rho_{A A}\left(a_{A A}-\bar{x}\right)+\rho_{A B}\left(a_{A B}-\bar{x}\right)+\rho_{B A}\left(a_{B A}-\bar{y}\right)+\rho_{B B}\left(a_{B B}-\bar{y}\right)$. Moreover,

$$
D=\bar{D}+V \text {. }
$$

Combining (4.2.11) and (4.2.12), we obtain $N / D \leqslant(\bar{N}+V \bar{N} / \bar{D}) /(\bar{D}+$ $V)=\bar{N} / \bar{D}$. Thus we have proved that $\sup _{\left(a_{k l}\right) \in \mathscr{A}} N / D \leqslant \bar{N} / \bar{D}=F\left(\rho_{A}\right)$. Optimising over $\left(\rho_{k l}\right) \in \mathscr{R}$, we arrive at $f \leqslant F\left(\rho^{*}(p)\right)$. Together with (4.2.9) this gives the claim.

(ii) Suppose that $\psi_{A B}(\bar{x})>\psi_{A A}(\bar{x})$ or $\psi_{B A}(\bar{y})>\psi_{B B}(\bar{y})$. Then, by choosing $a_{A A}=a_{A B}=\bar{x}, a_{B A}=a_{B B}=\bar{y}$ and $\rho_{A}=\rho^{*}(p)$ in (1.3.8), we get

$$
f>\frac{\rho^{*}(p) \bar{x} \psi_{A A}(\bar{x})+\left(1-\rho^{*}(p)\right) \bar{y} \psi_{B B}(\bar{y})}{\rho^{*}(p) \bar{x}+\left(1-\rho^{*}(p)\right) \bar{y}}=F\left(\rho^{*}(p)\right),
$$

where we use that $0<\rho^{*}(p)<1$ for all $0<p<p_{c}$ (recall Figure 6). 
4.2.2. Proof of Theorem 1.5.3. The following proposition is the analogue of Proposition 4.1.1.

Proposition 4.2.3. Fix $p<p_{c}$.

(i) $\partial \mathscr{D}$ lies on or below the supercritical curve $\alpha \mapsto \beta_{c}(\alpha)$.

(ii) $\left\{(\alpha, \alpha): \alpha \in\left[0, \alpha^{*}\right]\right\} \subset \partial \mathscr{D}$, where $\alpha^{*}$ is the same constant as for the supercritical curve, but lies below the diagonal elsewhere.

(iii) For every $p<p_{c}$ there exists an $\alpha^{*}(p) \in(0, \infty)$ such that the intersection of $\mathscr{D}$ with the lower half of $\mathrm{CONE}$ is the line segment $\{(\beta+$ $\left.\left.\alpha^{*}(p), \beta\right): \beta \in\left[-\frac{1}{2} \alpha^{*}(p), 0\right]\right\}$.

(iv) $\mathscr{D}$ is continuous and each line $\{(\beta+C, \beta): \beta \geqslant 0\}, C \in[-1,1)$, intersects $\partial \mathscr{D}$ in at most one point.

(v) As $p \downarrow 0$, the part of $\mathscr{D}$ not containing the diagonal segment of (ii) converges to the mirror image of the analytic continuation of the supercritical curve outside CONE. In particular, $\lim _{p \downarrow 0} \alpha^{*}(p)=\alpha_{0}$, with $\alpha_{0}$ the constant defined in the first line of (2.1.15).

(vi) $\lim _{p \uparrow p_{c}} \alpha^{*}(p)=\alpha_{1}$, with $\alpha_{1}$ the constant defined in the second line of (2.1.15).

P r o f. (i) Denote

$$
G(\mu, a)=\frac{1}{2}\left(\frac{\mu-1}{\mu}\right) \ln \left(\frac{a}{a-2}\right)+\frac{1}{\mu} \ln [2(a-1)] .
$$

Then Proposition 2.4.1 can be rewritten as the statement that $\psi_{A B}(a)>$ $\psi_{A A}(a)$ if and only if

$$
\phi^{\mathscr{J}}(\mu)>\frac{1}{2} \alpha+G(\mu, a) \quad \text { for some } \mu \geqslant 1,
$$

and similarly for $\psi_{B A}(a)>\psi_{B B}(a)$ with $\frac{1}{2} \beta$ instead of $\frac{1}{2} \alpha$. To prove the claim, we must show that, for all $(\alpha, \beta) \in \mathrm{CONE}$ and $p<p_{c}$, the following is true: For all $\mu \geqslant 1$,

$$
\phi^{\mathscr{I}}(\mu) \leqslant \frac{1}{2} \alpha+G(\mu, \bar{x}) \quad \text { and } \quad \phi^{\mathscr{J}}(\mu) \leqslant \frac{1}{2} \beta+G(\mu, \bar{y})
$$

imply $\phi^{\mathscr{F}}(\mu) \leqslant \frac{1}{2} \alpha+G\left(\mu, a^{*}\right)$. Indeed, by Theorem 1.5.2, Proposition 2.2.1, and Proposition 2.3.1, this yields that $(\alpha, \beta) \in \mathscr{D}$ for $p<p_{c}$ implies $(\alpha, \beta) \in \mathscr{D}$ for $p \geqslant p_{c}$.

We first show that the first half of (4.2.15) is redundant. Indeed,

$$
\begin{aligned}
& {\left[\frac{1}{2} \alpha+G(\mu, \bar{x})\right]-\left[\frac{1}{2} \beta+G(\mu, \bar{y})\right]=\frac{\alpha-\beta}{2}+[G(\mu, \bar{x})-G(\mu, \bar{y})]} \\
& \quad=-\frac{1}{2} \ln \left(\frac{\bar{x}(\bar{y}-2)}{\bar{y}(\bar{x}-2)}\right)+\frac{1}{2}\left(\frac{\mu-1}{\mu}\right) \ln \left(\frac{\bar{x}(\bar{y}-2)}{\bar{y}(\bar{x}-2)}\right)+\frac{1}{\mu} \ln \left(\frac{\bar{x}-1}{\bar{y}-1}\right) \\
& \quad=\frac{1}{2 \mu}\left[\ln \left(\frac{(\bar{x}-2)(\bar{x}-1)^{2}}{\bar{x}}\right)-\ln \left(\frac{(\bar{y}-2)(\bar{y}-1)^{2}}{\bar{y}}\right)\right] \geqslant 0,
\end{aligned}
$$


where in the second line we use the second line of (2.5.1), and in the last inequality we use that $\bar{x} \geqslant \bar{y}$ (recall Proposition 2.5.1(i)).

Thus, it remains to show that, for all $\mu \geqslant 1, \phi^{\mathscr{J}}(\mu) \leqslant \frac{1}{2} \beta+G(\mu, \bar{y})$ implies $\phi^{\mathscr{J}}(\mu) \leqslant \frac{1}{2} \alpha+G\left(\mu, a^{*}\right)$. Indeed,

$$
\begin{aligned}
& {\left[\frac{1}{2} \alpha+G\left(\mu, a^{*}\right)\right]-\left[\frac{1}{2} \beta+G(\mu, \bar{y})\right]=\frac{\alpha-\beta}{2}+\left[G\left(\mu, a^{*}\right)-G(\mu, \bar{y})\right] } \\
&=- \frac{1}{2} \ln \left(\frac{\bar{x}(\bar{y}-2)}{\bar{y}(\bar{x}-2)}\right)+\frac{1}{2}\left(\frac{\mu-1}{\mu}\right) \ln \left(\frac{a^{*}(\bar{y}-2)}{\bar{y}\left(a^{*}-2\right)}\right)+\frac{1}{\mu} \ln \left(\frac{a^{*}-1}{\bar{y}-1}\right) \\
&= \frac{1}{2}\left[\ln \left(\frac{a^{*}}{a^{*}-2}\right)-\ln \left(\frac{\bar{x}}{\bar{x}-2}\right)\right] \\
& \quad+\frac{1}{2 \mu}\left[\ln \left(\frac{\left(a^{*}-2\right)\left(a^{*}-1\right)^{2}}{a^{*}}\right)-\ln \left(\frac{(\bar{y}-2)(\bar{y}-1)^{2}}{\bar{y}}\right)\right] \geqslant 0,
\end{aligned}
$$

where in the second line we use the second line of (2.5.1), and in the last inequality we use that $\bar{x} \geqslant a^{*} \geqslant \bar{y}$ (recall Proposition 2.5.1(i)) to get that both terms between square brackets are $\geqslant 0$.

$\mathrm{R} \mathrm{e} \mathrm{m}$ a r k. The redundancy of the first half of (4.2.15) shows that, as the critical curve is crossed from $\mathscr{D}$ to $\mathscr{L}$, localization occurs in the $B A$-blocks rather than in the $A B$-blocks. This is why the first criterion in Proposition 4.2.2(i) is redundant (as was claimed in (1.5.7)).

(ii) If $\alpha=\beta$, then $\bar{x}=\bar{y}=a^{*}$ by Proposition 2.5.1(ii). Therefore the second criterion in Proposition 4.2.2(i) reduces to $\psi_{B A}\left(a^{*}\right)=\psi_{B B}\left(a^{*}\right)$ (while the first criterion in Proposition 4.2.2(i) is redundant). But if $\alpha=\beta$, then $\psi_{B A}=\psi_{A B}$ and $\psi_{B B}=\psi_{A A}$. Hence, the criterion for delocalization on the diagonal reads $\psi_{A B}\left(a^{*}\right)=\psi_{A A}\left(a^{*}\right)$, which is the same as the criterion for delocalization in the supercritical case (recall Proposition 2.2.1 and 4.1.1).

This also shows that $\partial \mathscr{D}$ must leave the diagonal at the same point as the supercritical curve, i.e., at $\left(\alpha^{*}, \alpha^{*}\right)$. (Incidentally, note that $\alpha=\beta$ does not imply $\psi_{B A}=\psi_{B B}$ or $\psi_{A B}=\psi_{A A}$, because only matches of the polymer and the emulsion receive an energy.)

(iii) Let $\alpha \geqslant 0$ and $\beta \leqslant 0$. By (2.2.13), $\phi^{\mathscr{J}}(\mu ; \alpha, \beta)=\frac{1}{2} \alpha+\widehat{\kappa}(\mu)$. It therefore follows from (1.5.7) and the line below (4.2.14) that $(\alpha, \beta) \notin \mathscr{D}$ if and only if

$$
\exists \mu \geqslant 1: \quad \widehat{\kappa}(\mu)>G(\mu, \bar{y})-\frac{1}{2} C,
$$

where we recall from Section 2.5 that $\bar{y}$ is a function of $C=\alpha-\beta$ and $\rho^{*}(p)$ only. Combining the second line of (2.5.1) with (4.2.13), we have

$$
\begin{aligned}
G(\mu, \bar{y})-\frac{1}{2} C & =\frac{1}{2}\left(\frac{\mu-1}{\mu}\right) \ln \left(\frac{\bar{y}}{\bar{y}-2}\right)+\frac{1}{\mu} \ln [2(\bar{y}-1)]-\frac{1}{2} C \\
& =-\frac{1}{2 \mu} \ln \left(\frac{\bar{y}}{\bar{y}-2}\right)+\frac{1}{\mu} \ln [2(\bar{y}-1)]+\frac{1}{2} \ln \left(\frac{\bar{x}}{\bar{x}-2}\right) .
\end{aligned}
$$


By Proposition 2.5.1(iv), the right-hand side of (4.2.17) is strictly decreasing in $C$ for fixed $\mu$ and $\rho^{*}(p)$. Hence there is a unique critical value $C^{*}$, which we call $\alpha^{*}(p)$, above which (4.2.16) holds. Since $\bar{x} \uparrow \infty$ and $\bar{y} \downarrow 2$ as $C \rightarrow \infty$ by Proposition 2.5.1(vii), the right-hand side tends to $-\infty$ as $-(1 / 2 \mu)[\ln (1 / 2(\bar{y}-2))+o(1)]+o(1)$. Since $\widehat{\kappa}(\mu) \geqslant 0$ for all $\mu \geqslant 1$, it follows that $C^{*}$ is finite.

(iv) The continuity of $\mathscr{D}$ is immediate from Lemma 2.2.3, Proposition 2.5.1(iv), Proposition 2.5.1, and Proposition 4.2.2.

To prove the remainder of the claim, we need the following.

Lemma 4.2.1. $\beta \mapsto \phi^{\mathscr{J}}(\mu ; \beta+C, \beta)-\frac{1}{2} \beta$ is nondecreasing on $[0, \infty)$ for all $\mu \geqslant 1$ and $C \geqslant 0$.

$\mathrm{P}$ r o of. The function $(\alpha, \beta) \mapsto \phi^{\mathscr{\sigma}}(\alpha, \beta ; \mu)$ is convex on $\mathbf{R}^{2}$ for all $\mu \geqslant 1$, by an argument similar to that given in the proof of Theorem 1.3.1(ii) in Section 3.1 (recall (2.2.3)-(2.2.5) and (2.2.10)). Fix $\mu \geqslant 1$ and $C=$ $\alpha-\beta \geqslant 0$. Denote $\Delta(\beta)=\phi^{\mathscr{J}}(\beta+C, \beta ; \mu)-\frac{1}{2} \beta$. Then $\beta \mapsto \Delta(\beta)$ is convex. Moreover, by $(2.2 .13), \Delta(0)=\phi^{\mathscr{J}}(C, 0 ; \mu)=\frac{1}{2} C+\widehat{\kappa}(\mu)$ and $\Delta(\beta) \geqslant$ $\frac{1}{2}(\beta+C)+\widehat{\kappa}(\mu)-\frac{1}{2} \beta=\Delta(0)$ for $\beta \geqslant 0$. Hence $\beta \mapsto \Delta(\beta)$ is nondecreasing on $[0, \infty)$.

To prove the claim, use that $\bar{x}$ and $\bar{y}$ are functions of $C=\alpha-\beta$ only, repeat the same argument as in the proof of (iii), and use Lemma 4.2.1.

(v) The limit $p \downarrow 0$ corresponds to $\rho \downarrow 0$ (recall Figure 6). By Proposition 2.5.1(vi), $\bar{y} \uparrow a^{*}$ when $\alpha<\beta+\ln 5$ and $\bar{y} \uparrow 2 /\left(1-e^{-(\alpha-\beta)}\right)$ when $\alpha \geqslant \beta+\ln 5$. In the first case, the criterion for delocalization reduces to $\sup _{\mu \geqslant 1} \mu\left[\phi^{\mathscr{J}}(\mu)-\frac{1}{2} \beta-\frac{1}{2} \ln 5\right] \leqslant \frac{1}{2} \ln \frac{9}{5}$. Recalling Propositions 2.2.1 and 2.3.1, we see that this is precisely the criterion for delocalization in the supercritical case but with $\alpha$ and $\beta$ interchanged. The second case is ruled out by the observation that $\alpha_{0} \leqslant \ln 5$, as is immediate from the first line of (2.1.15).

(vi) The limit $p \uparrow p_{c}$ corresponds to $\rho \uparrow 1$. Let $\beta=0$. Then, by Proposition 2.5.1(v), $\bar{y} \downarrow 10 /\left(5-e^{-\alpha}\right)$ as $\rho \uparrow 1$. Therefore, with the help of Proposition 2.4.1 and (2.2.13), the criterion for delocalization when $\alpha \geqslant 0$ reduces to

$$
\sup _{\mu \geqslant 1} \mu\left[\widehat{\kappa}(\mu)-\frac{1}{2} \ln 5\right] \leqslant \frac{1}{2} \ln \left[\frac{4 e^{-\alpha}\left(5+e^{-\alpha}\right)^{2}}{5\left(5-e^{-\alpha}\right)^{2}}\right] .
$$

This inequality holds if and only if $\alpha \leqslant \alpha_{1}$.

The redundancy of the first half of (4.2.15), which is strict when $\alpha>\beta$, shows that, as the critical curve is crossed from $\mathscr{D}$ to $\mathscr{L}$ off the diagonal, localization occurs in the $B A$-blocks rather than in the $A B$-blocks.

4.2.3. Full phase diagram. With the help of the two symmetry properties stated in (1.3.9), the phase diagram can be extended from CONE to $\mathbf{R}^{2}$. When doing so, we obtain the full phase diagram given in Figures 9 and 10. Here the label on $\mathscr{D}(\mathscr{L})$ indicates the type of (de)localization. 
We next explain why in Figures 9 and 10 the diagonal segment $\{(\alpha, \alpha)$ : $\left.\alpha \in\left[-\alpha^{*}, \alpha^{*}\right]\right\}$ is a critical line. This is not obvious from our earlier considerations, because the segment lies on the boundary of CONE.

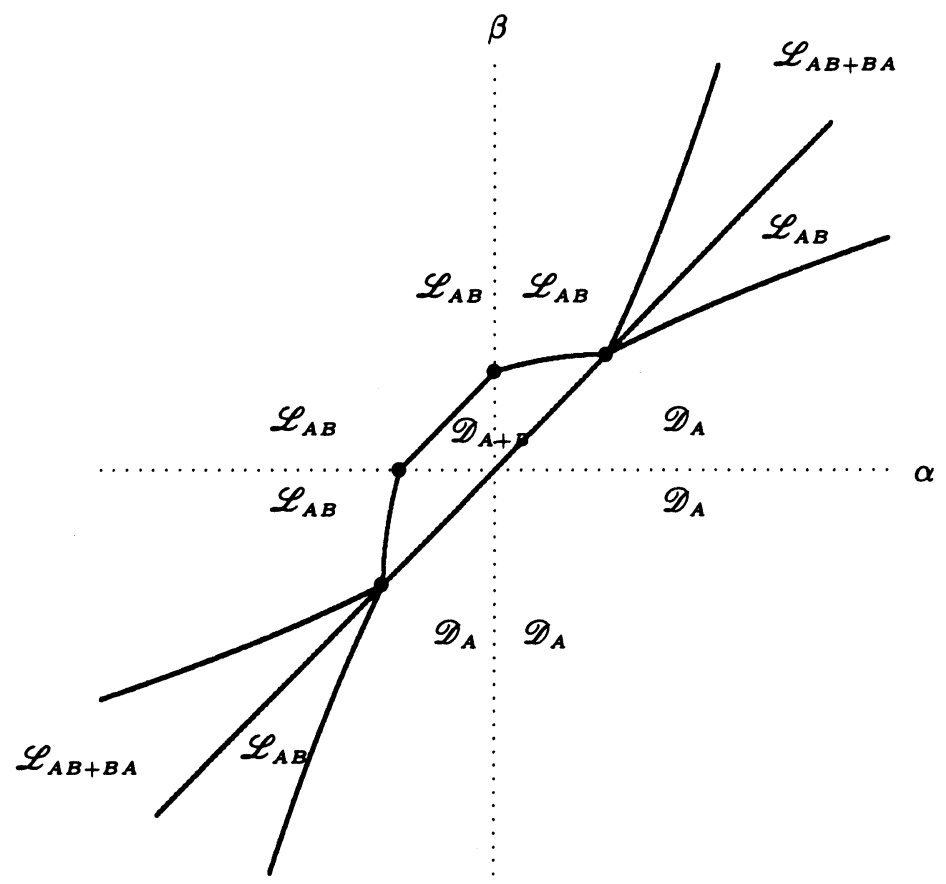

Fig. 9. Full phase diagram for $p \geqslant p_{c}$.

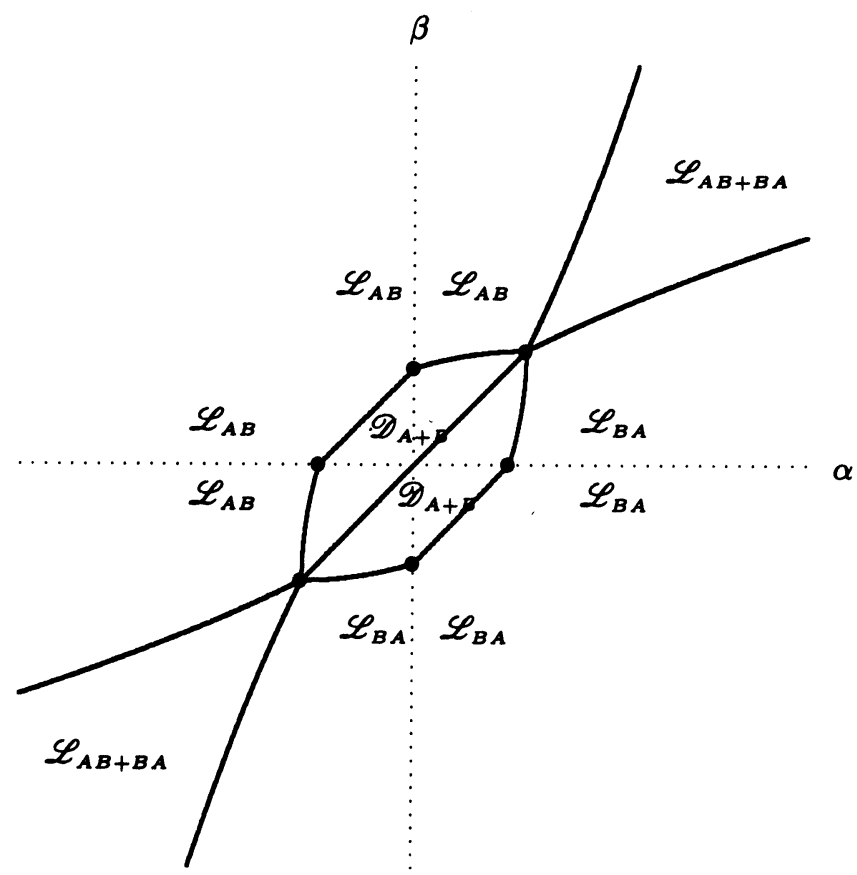

Fig. 10. Full phase diagram for $1-p_{c}<p<p_{c}$. 
Take Figure $9\left(p \geqslant p_{c}\right)$. We have $f=\psi_{A A}\left(a^{*}\right)$ in the phase $\mathscr{D}_{A}$ and $f=\psi_{A A}(\bar{y})$ in the phase $\mathscr{D}_{A+B}$, where $\bar{y}=\bar{y}\left(\beta, \alpha ; \rho^{*}(1-p)\right)$ is the $y$ maximizer of (1.5.3) with $\alpha \leftrightarrow \beta$ and $p \leftrightarrow 1-p$. Since $a^{*}$ is the unique maximizer of $\psi_{A A}$ (by Lemma 2.1.1(iv) and (2.2.2)) and $\bar{y} \neq a^{*}$ when $\beta>\alpha$ (by Proposition 2.5.1(i)), this shows that the free energy is nonanalytic along the separation line between $\mathscr{D}_{A}$ and $\mathscr{D}_{A+B}$.

Take Figure $10\left(1-p_{c}<p<p_{c}\right)$. We have $f=F\left(\alpha, \beta ; \rho^{*}(p)\right)$ in the lower half of the phase $\mathscr{D}_{A+B}$ and $f=F\left(\beta, \alpha ; \rho^{*}(1-p)\right)$ in the upper half of the phase $\mathscr{D}_{A+B}$. Similarly as in Theorem 1.3.1(iv), there is the symmetry $F\left(\beta, \alpha ; \rho^{*}(1-p)\right)=F\left(\alpha, \beta ; 1-\rho^{*}(1-p)\right)$ (as is also obvious from (1.5.3) and (2.5.1)). However, $\rho^{*}(p)>1-\rho^{*}(1-p)$, for any $p \in(0,1)$ since the curve in Figure 6 lies strictly above the diagonal. Since $\rho \mapsto F(\alpha, \beta ; \rho)$ is strictly increasing (as we saw below (4.2.8)), this shows that free energy is nonanalytic along the separation line between the two halves of $\mathscr{D}_{A+B}$.

It might be that in Figure 10 also inside the two phases $\mathscr{L}_{A B+B A}$ there is a critical line on the diagonal. We do not expect this, but we lack the tools to decide.

4.3. Further observations about $\mathscr{L}$ for $p<p_{c}$. We close by making some observations about the second subcritical curve, lying inside $\mathscr{L}$. By Proposition 4.2.2(ii), the criterion for $\mathscr{L}$ is $\psi_{B A}(\bar{y})>\psi_{B B}(\bar{y})$, corresponding to $B A$-localization. The phase $\mathscr{L}$ splits further into two parts, one where $A B$-localization does not occur and one where it does. The criterion for $A B$-localization reads

$$
\psi_{A B}(\widetilde{x})>\psi_{A A}(\widetilde{x}),
$$

where we denote by $\tilde{x}, \tilde{y}, \tilde{z}$ the values of the minimizers $a_{A B}\left(=a_{A A}\right)$, $a_{B A}, a_{B B}$, respectively, when in the variational expression for the free energy (1.3.8) we replace $\psi_{A B}$ by $\psi_{A A}$. Indeed, this is in complete analogy with the argument in Section 4.2.1 identifying the first subcritical curve as the one arising when in the variational expression for the free energy (1.3.8) we replace $\psi_{A B}$ by $\psi_{A A}$ and $\psi_{B A}$ by $\psi_{B B}$. Unfortunately, whereas the latter reduction leads to a computable supremum (as shown in Section 4.2.1), the former reduction does not (because we have no closed form expression for $\psi_{B A}$ ). Consequently, we have little information on $\widetilde{x}, \widetilde{y}, \widetilde{z}$ (unlike for $\bar{x}, \bar{y}$ ), which is why (4.3.1) is hard to exploit. However, we can use (4.3.1) to obtain a lower bound on the second subcritical curve.

Lemma 4.3.1. If $\alpha \geqslant 0$ and $\beta \leqslant \ln \left(2-e^{-\alpha}\right)$, then $\psi_{A B} \equiv \psi_{A A}$ and hence (4.3.1) fails.

P r o o f. From (4.1.6) we know that if $\beta \leqslant \ln \left(2-e^{-\alpha}\right)$, then $\phi^{\mathscr{I}}(\mu) \leqslant$ $\alpha / 2+\widehat{\kappa}(\mu)$ and hence

$$
\sup _{\mu \geqslant 1} \mu\left[\phi^{\mathscr{I}}(\mu)-\frac{\alpha}{2}-\frac{1}{2} \ln \left(\frac{a}{a-2}\right)\right] \leqslant \sup _{\mu \geqslant 1} \mu\left[\widehat{\kappa}(\mu)-\frac{1}{2} \ln \left(\frac{a}{a-2}\right)\right] .
$$


But

$$
\sup _{\mu \geqslant 1} \mu\left[\widehat{\kappa}(\mu)-\frac{1}{2} \ln \left(\frac{a}{a-2}\right)\right] \leqslant \frac{1}{2} \ln \left[\frac{4(a-2)(a-1)^{2}}{a}\right],
$$

as can be seen from Proposition 2.4.1, because $\phi^{\mathscr{I}} \equiv \widehat{\kappa}$ and $\psi_{A B} \equiv \psi_{A A}$ when $\alpha=\beta=0$. Combining (4.3.2) and (4.3.3) with Proposition 2.4.1, we find that if $\beta \leqslant \ln \left(2-e^{-\alpha}\right)$, then $\psi_{A B} \equiv \psi_{A A}$.

Lemma 4.3.1 shows that the second critical curve is bounded below by the curve $\alpha \mapsto \ln \left(2-e^{-\alpha}\right)$.

The second subcritical curve splits off the diagonal at the same point $\left(\alpha^{*}, \alpha^{*}\right)$ as the first subcritical curve. Indeed, if $\alpha=\beta$, then $\psi_{A B} \equiv \psi_{B A}, \psi_{A A} \equiv \psi_{B B}$, and $\bar{x}=\bar{y}$. Therefore, on the diagonal the criteria for $A B$-localization $\left(\psi_{A B}\left(a^{*}\right)=\psi_{A A}\left(a^{*}\right)\right)$ and $B A$-localization $\left(\psi_{B A}\left(a^{*}\right)=\psi_{B B}\left(a^{*}\right)\right)$ coincide.

We believe that the second critical curve has a finite horizontal asymptote, as argued on physical grounds in Section 1.6, and that it lies above the supercritical curve. We are unable to prove this.

\section{REFERENCES}

1. Albeverio $S$., Zhou $X$. Y. Free energy and some sample path properties of a random walk with random potential. - J. Stat. Phys., 1996, v. 83, № 3-4, p. 573-622.

2. Biskup M., den Hollander F. A heteropolymer near a linear interface. - Ann. Appl. Probab., 1999, v. 9, № 3, p. 668-687.

3. Bodineau T., Giacomin G. On the localization transition of random copolymers near selective interfaces. - J. Stat. Phys., 2004, v. 117, № 5-6, p. 801-818.

4. Bolthausen $E$., den Hollander $F$. Localization transition for a polymer near an interface. - Ann. Probab., 1997, v. 25, № 3, p. 1334-1366.

5. Brazhnyi V.A., Stepanow S. Adsorption of a random heteropolymer with random self-interactions onto an interface. - Eur. Phys. J. B - Condensed Matter, 2002, v. 27 , № 3, p. $355-362$.

6. Caravenna F., Giacomin G., Gubinelli M. A numerical approach to copolymers at selective interfaces. - J. Stat. Phys. (to appear).

7. Causo M.S., Whittington S. G., A Monte Carlo investigation of the localization transition in random copolymers at an interface. - J. Phys. A: Math. Gen., 2003, v. 36, № 13, p. L189-L195.

8. Comets $F$. Large deviation estimates for a conditional probability distribution. Applications to random interaction Gibbs measures. - Probab. Theory Relat. Fields, 1989 , v. 80, № 3, p. 407-432.

9. Garel T., Huse D. A., Leibler $S$., Orland $H$. Localization transition of random chains at interfaces. - Europhys. Lett., 1989, v. 8, p. 9-13.

10. Garel T., Monthus $C$. Two-dimensional wetting with binary disorder: a numerical study of the loop statistics. - Eur. Phys. J. B - Condensed Matter, 2005, v. 46, № 1, p. 117-125.

11. Giacomin G., Toninelli F. L. Estimates on path delocalization for copolymers at selective interfaces. - Probab. Theory Relat. Fields, 2005, v. 133, № 4, p. 464-482.

12. Giacomin G., Toninelli F. L. Smoothing effect of quenched disorder on polymer depinning transitions. - Commun. Math. Phys. (to appear).

13. Giacomin G., Toninelli F. L. The localized phase of disordered copolymers with adsorption. - http://www.arxiv.org/math.PR/0510047. 
14. Greven A., den Hollander $F$. Branching random walk in random environment: phase transitions for local and global growth rates. - Probab. Theory Relat. Fields, 1992, v. 91 , № 2, p. 195-249.

15. Grosberg A., Izrailev S., Nechaev $S$. Phase transition in a heteropolymer chain at a selective interface. - Phys. Rev. E, 1994, v. 50, № 3, p. 1912-1921.

16. den Hollander F. Large Deviations, Fields Institute Monograph Series. Providence: AMS, 2000, $143 \mathrm{p}$.

17. den Hollander $F$., Wüthrich $M$. Diffusion of a heteropolymer in a multi-interface medium. - J. Stat. Phys., 2004, v. 114, p. 849-889.

18. James E. W., Soteros C. E., Whittington S. G. Localization of a random copolymer at an interface: an exact enumeration study. - J. Phys. A: Math. Gen., 2003, v. 36, № 46, p. 11575-11584.

19. Janse van Rensburg E. J., Orlandini E., Tesi M. C., Whittington S. G. Self-averaging in random self-attracting polygons. - J. Phys. A: Math. Gen., 2001, v.34, №6, p. L37-L44.

20. Madras N., Whittington S.G. Self-averaging in finite random copolymers. J. Phys. A: Math. Gen., 2002, v. 35, № 28, p. L427-L431.

21. Madras N., Whittington S. G. Localization of a random copolymer at an interface. J. Phys. A: Math. Gen., 2003, v. 36, № 4, p. 923-938.

22. Maritan A., Riva M. P., Trovato A. Heteropolymers in a solvent at an interface. J. Phys. A: Math. Gen., 1999, v. 32, № 32, p. L275-L280.

23. Martin R., Causo M.S., Whittington S. G. Localization transition for a randomly coloured self-avoiding walk at an interface. - J. Phys. A: Math. Gen., 2000, v. 33, № 44, p. $7903-7918$.

24. Monthus $C$. On the localization of random heteropolymers at the interface between two selective solvents. - Eur. Phys. J. B - Condensed Matter, 2000, v. 13, № 1, p. $111-130$.

25. Orlandini E., Rechnitzer A., Whittington S. G. Random copolymers and the Morita approximation: polymer adsorption and polymer localization. - J. Phys. A: Math. Gen., 2002, v. 35, № 36, p. 7729-7751.

26. Orlandini E., Tesi M.C., Whittington S.G. A self-avoiding walk model of random copolymer adsorption. - J. Phys. A: Math. Gen., 1999, v. 32, №3, p. 469-477.

27. Orlandini E., Tesi M. C., Whittington S. G. Self-averaging in models of random copolymer collapse. - J. Phys. A: Math. Gen., 2000, v. 33, № 2, p. 259-266.

28. Seppäläinen T. Entropy, limit theorems, and variational principles for disordered lattice systems. - Commun. Math. Phys., 1995, v. 171, № 2, p. 233-277.

29. Синай Я.Г. Случайное блуждание со случайным потенциалом. - Теория вероятн. и ее примен., 1993 , т. 38 , в. 2 , с. $457-460$.

30. Soteros C.E., Whittington S. G. The statistical mechanics of random copolymers. J. Phys. A: Math. Gen., 2004, v. 37, № 41, p. R279-R325.

31. Stepanow S., Sommer J.-U., Erukhimovich I. Ya. Localization transition of random copolymers at interfaces. - Phys. Rev. Lett., 1998, v. 81, № 20, p. 4412-4415.

32. Trovato A., Maritan A. A variational approach to the localization transition of heteropolymers at interfaces. - Europhys. Lett., 1999, v. 46, p. 301-306.

33. Whittington S. G. Random copolymers. - Physica A, 2002, v. 314, № 1-4, p. 214-219. 